\title{
Topological confinement of vortices in two-flavor dense QCD
}

\author{
Yuki Fujimoto $^{a}$ and Muneto Nitta ${ }^{b}$ \\ ${ }^{a}$ Department of Physics, The University of Tokyo, \\ 7-3-1 Hongo, Bunkyo-ku, Tokyo 113-0033, Japan \\ ${ }^{b}$ Department of Physics $\&$ Research and Education Center for Natural Sciences, Keio University, \\ Hiyoshi 4-1-1, Yokohama, Kanagawa 223-8521, Japan \\ E-mail: fujimoto@nt.phys.s.u-tokyo.ac.jp, nitta@phys-h.keio.ac.jp
}

ABSTRACT: We find a novel confinement mechanism in the two-flavor dense quark matter proposed recently, that consists of the 2SC condensates and the $P$-wave diquark condensates of $d$-quarks. This quark matter exhibiting color superconductivity as well as superfluidity is classified into two phases; confined and deconfined phases of vortices. We establish that the criterion of the confinement is color neutrality of Aharonov-Bohm (AB) phases: vortices exhibiting color non-singlet $\mathrm{AB}$ phases are confined by the so-called $\mathrm{AB}$ defects to form color-singlet bound states. In the deconfined phase, the most stable vortices are non-Abelian Alice strings, which are superfluid vortices with fractional circulation and non-Abelian color magnetic fluxes therein, exhibiting color non-singlet AB phases. On the other hand, in the confined phase, these non-Abelian vortices are confined to either a baryonic or mesonic bound state in which constituent vortices are connected by AB defects. The baryonic bound state consists of three non-Abelian Alice strings with different color magnetic fluxes with the total flux canceled out connected by a domain wall junction, while the mesonic bound state consists of two non-Abelian Alice strings with the same color magnetic fluxes connected by a single domain wall. Interestingly, the latter contains a color magnetic flux in its core, but this can exist because of color neutrality of its AB phase.

KEYwords: Solitons Monopoles and Instantons, Phase Diagram of QCD, Spontaneous Symmetry Breaking

ARXIV EPrINT: 2103.15185 


\section{Contents}

1 Introduction $\quad 2$

2 Two-flavor dense quark matter 4

$2.12 \mathrm{SC}+\langle d d\rangle$ phase $\quad 5$

2.2 Symmetry of the $2 \mathrm{SC}+\langle d d\rangle$ phase: general consideration 6

2.3 Symmetry breaking by the $\langle d d\rangle$ condensate $\quad 7$

2.4 Symmetry breaking by the 2SC condensate 8

2.5 The opposite ordering of symmetry breakings 9

$\begin{array}{lll}2.6 & \text { The overall symmetry breaking } G \rightarrow K & 10\end{array}$

3 Topological vortices in $\langle d d\rangle$ phase $\quad 10$

$\begin{array}{ll}3.1 \text { Abelian superfluid vortices } & 10\end{array}$

$\begin{array}{lll}3.2 & \text { Non-Abelian Alice strings } & 11\end{array}$

$\begin{array}{lll}3.3 & \text { Doubly-wound non-Abelian strings } & 12\end{array}$

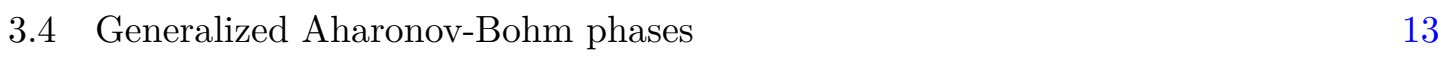

4 Vortex confinement $\quad \mathbf{1 5}$

4.1 Aharonov-Bohm defects in 2SC condensate $\Phi_{2 \mathrm{SC}} \quad 15$

$\begin{array}{ll}4.2 \text { Baryonic molecule } & 17\end{array}$

$\begin{array}{lll}4.3 & \text { Mesonic molecule } & 18\end{array}$

4.4 Baryons-to-mesons decay 20

5 Consistency with the opposite ordering in the symmetry breaking 20

$\begin{array}{lll}5.1 & \text { Superfluid vortex } & 20\end{array}$

$\begin{array}{lll}5.2 \mathrm{U}(1)_{\mathrm{C}+\mathrm{B}} \text { vortices } & 21\end{array}$

$6 \quad$ Summary and discussions $\quad 22$

A Pure color flux tubes $\quad 24$

B Generalized Aharonov-Bohm phases around vortices $\quad 24$

B.1 Aharonov-Bohm phase around Abelian superfluid strings 24

B.2 Aharonov-Bohm phase around pure color flux tubes 25

$\begin{array}{lll}\text { B.2.1 Pure color flux tube(d) } & 25\end{array}$

B.2.2 Pure color flux tube(c) 26

B.3 Aharonov-Bohm phase around non-Abelian Alice strings 27

B.4 Aharonov-Bohm phase around doubly-wound non-Abelian strings 28 


\section{Introduction}

Color confinement is one of the most challenging unsolved problems in modern high energy physics. Particles belonging to non-singlet representations of color gauge group SU(3), such as quarks and gluons, cannot exist alone. Instead, they are confined to form colorsinglet composite particles, that is, hadrons such as baryons and mesons. The dual Meissner picture suggests that color electric fluxes emanating from quarks are squeezed to flux tubes and confine these quarks, as a dual to the Meissner effect: magnetic fluxes emanating from monopoles are squeezed to flux tubes or vortices and confine these monopoles [1, 2].

For QED in $2+1$ dimensions, a duality maps particles confined by strings to vortices confined by domain wall strings [3]. There are several models exhibiting a vortex confinement, that is, vortices are linearly confined by solitons, domain walls, or kinks stretching among (between) them. Bose-Einstein condensates of multiple components serve as test beds for a vortex confinement; they allow fractional vortices in the system, which are confined when intercomponent (Rabi or Josephson) couplings are introduced. Various facets of this model have been known in details: although dimensionality is different from QCD, similarities with quark confinement were pointed out [4] and were studied extensively [5-9]. Phase diagram of vortex confinement phase transitions was also determined [10]. Two-gap superconductors also offer another circumstance where fractional vortices [11] are confined by domain lines [12, 13], and vortex confinement was discussed [14]. In $3+1$ dimensions, these configurations are vortex strings confined by domain walls (membranes), but they can be applied to confinement of QCD-like theory on a compactified circle [15]. Another mechanism responsible for vortex confinement is given in the so-called modified XY model [16-18] in which the modified gradient term with half periodicity compared with the canonical gradient term plays an essential role. More recently, a novel vortex confinement mechanism based on topology was proposed; when some fields receiving nontrivial Aharonov-Bohm (AB) phases around a vortex develop vacuum expectation values (VEVs), there must appear kinks or domain walls attached to the vortex in order to compensate the $\mathrm{AB}$ phases to maintain singlevaluedness of the VEVs [19-21]. We referred such defects AB defects. We may call this confinement as "topological confinement."

In this paper, we show that such a topological confinement of vortices occurs in the ground state of cold QCD matter at high baryon densities, which exhibits color superconductivity [22-24]. Among various known color-superconducting phases, the color-flavor locked (CFL) phase [25] in three-flavor symmetric matter is realized at extremely high density limit while the 2-flavor superconducting (2SC) phase [26, 27] appears at relatively low density where strange quark mass cannot be neglected. For the case of three-flavor symmetric matter, the symmetry breaking patterns and low-energy excitations such as corresponding Nambu-Goldstone modes have one-to-one correspondence between both phases; this observation led to the concept of quark-hadron continuity that posit the continuous connection between quark matter (CFL color superconductor) and hadronic matter (hyperonic superfluid) [28]. Quark-hadron continuity with three-flavor symmetry has been well understood [28-34] (see, however, refs. [35, 36] for recent works on quark-hadron continuity in the presence of vortices, which is at variance with the conventional scenario). It has also been repurposed for the modern way of constructing the equation of state of 
neutron stars with quark cores [37-41]. For the two-flavor symmetric case, the similar quark-hadron continuity was thought to be absent. However, it was pointed out recently that the additional diquark pairing between $d$-quarks $\left\langle\hat{d}^{\top} \mathcal{C} \gamma^{i} \nabla^{j} \hat{d}\right\rangle$ (which we often denote by $\langle d d\rangle$ for simplicity) can arise in the ${ }^{3} P_{2}$ channel besides the conventional 2SC condensate $[42,43]$, and this novel quark phase named $2 \mathrm{SC}+\langle d d\rangle$ phase opened the possibility for continuity to the ${ }^{3} P_{2}$ neutron superfluid phase [44-55]. This paper particularly focuses on this $2 \mathrm{SC}+\langle d d\rangle$ phase for a topological vortex confinement.

Quantum vortices, that is vortices with quantized circulations, are essential degrees of freedom in superfluids. For instance, when superfluids are rotating rapidly, quantum vortices are created along the rotation axis to form a vortex lattice. Such the quantum vortices or color magnetic flux tubes are also present in color-superconducting quark matter [56]. In the CFL phase, Abelian superfluid vortices exist as the topologically stable configuration due to the nontrivial homotopy group $\pi_{1}\left[\mathrm{U}(1)_{\mathrm{B}}\right]=\mathbb{Z}[57,58]$. However, this is unstable against a decay into a triad of more stable vortices [59-61], which are non-Abelian vortices with color magnetic fluxes and fractional circulation of the Abelian vortices [56, 59, 62-64]. A single non-Abelian vortex carries the so-called orientational moduli (collective coordinates) of the complex projective space $\mathbb{C} P^{2}$ as Nambu-Goldstone modes trapped in its core $[56,59,65,66]$ as well as a triplet of gapless Majorana fermions [67, 68]. Recently, in the context of quark-hadron continuity, vortices penetrating through the CFL phase into hyperon matter have been extensively discussed [35, 36, 69-73] (see also ref. [74] for the similar attempt in two-flavor setup). Vortex confinement in the CFL phase has been proposed recently [75] as a non-Abelian generalization of two-component BECs and two-gap superconductors, but this is not a topological vortex confinement.

On the other hand, the pure 2SC phase, which can be regarded as more realistic than the CFL phase in the sense that strange quark mass is not degenerate with light quark masses, cannot support topologically stable vortices because of the unbroken $\mathrm{U}(1)_{\mathrm{B}}$ symmetry resulting in a trivial first homotopy group [76]. However, the $2 \mathrm{SC}+\langle d d\rangle$ phase admits stable topological vortices that winds around the $\langle d d\rangle$ condensate [77]. This $2 \mathrm{SC}+\langle d d\rangle$ phase can further be subdivided into at least two kinds of phases, deconfined and confined phases depending on the form of the $2 \mathrm{SC}$ condensate, i.e., if it is restricted to real value then the phase falls into the deconfined one, and if it is complex-valued then the confined phase is realized. In the preceding paper [77], we only concentrated on the deconfined phase in which we assumed that value of the $2 \mathrm{SC}$ condensate can be taken to be real. In the deconfined phase, the most stable vortices are non-Abelian Alice vortices, which carries a $1 / 3$ fractional circulation in $\mathrm{U}(1)_{\mathrm{B}}$ and the color-magnetic fluxes. A single non-Abelian Alice string is accompanied by orientational moduli (collective coordinates) of the real projective space $\mathbb{R} P^{2}$ corresponding to the color flux therein in the presence of the $\langle d d\rangle$ condensates alone. In these respects, the non-Abelian Alice strings are similar to the CFL non-Abelian vortices. At the same time there are features unique to the non-Abelian Alice strings such as topological obstruction. It means that some unbroken generators in the bulk are not globally defined around the string, akin to Alice strings [19, 78-86]. Another unique feature is that quarks receive color non-singlet (generalized) AB phases when they encircle the strings, in contrast to the CFL case where non-Abelian vortices obtain only 
color-singlet AB phases. Here, we have called the phase without the baryon circulation as (pure) $\mathrm{AB}$ phase and the one with the baryon circulation as generalized AB phase. The former is relevant for heavy quarks while the latter is necessary for light quarks $u$ and $d$ because they participate in the condensations $\langle d d\rangle$ with a vortex configuration. As a consequence of the nontrivial AB phases, the "bulk-soliton moduli locking" occurs in the deconfined phase, i.e., the 2SC condensates develop VEVs whose color fluxes are enforced to be aligned along the orientational moduli $\mathbb{R} P^{2}$ of the Alice string because of the singlevaluedness of the VEVs [77]. Due to superfluidity of the system, two separated vortices repel each other and no bound states are formed in the deconfined phase. Thus, a single Abelian $\mathrm{U}(1)_{\mathrm{B}}$ vortex is unstable against a decay into a triad of non-Abelian Alice strings with total color canceled out among these three.

In this paper, we show that in the confined phase, these non-Abelian vortices are confined to either a baryonic or mesonic bound state in which constituent vortices are connected by $\mathrm{AB}$ defects [19-21] that appear to compensate nontrivial color non-singlet (generalized) $\mathrm{AB}$ phases of the $2 \mathrm{SC}$ condensates around vortices. The baryonic bound state consists of three non-Abelian Alice strings with different color magnetic fluxes with the total flux canceled out, which are connected by a domain wall junction. Since the domain walls pull vortices by their tension, these vortices are combined and result in a single Abelian $\mathrm{U}(1)_{\mathrm{B}}$ vortex. On the other hand, the mesonic bound state consists of two non-Abelian Alice strings with the same color magnetic fluxes connected by a single domain wall. Again, they are pulled by the domain wall tension and result in a doublywound non-Abelian string. Interestingly, the latter still contains a color magnetic flux in its core. Nevertheless, this is screened at large distances, since it has only a color-singlet $\mathrm{AB}$ phase. Thus, we establish that color neutrality of the $\mathrm{AB}$ phases is the criterion of the confinement of vortices.

Baryonic-type molecules of vortices can be found in three-component BECs [87], threegap superconductors [88], and the $\mathbb{Z}_{3}$ modified XY model [18]. Baryons in this paper look similar to these configurations, but a crucial difference lies in that baryons in this paper are $\mathrm{SU}(3)_{\mathrm{C}}$ color singlets.

The outline of the paper is as follows. In section 2 , we summarize the $2 \mathrm{SC}+\langle d d\rangle$ phase with paying a particular attention to the confined phase. In section 3, we introduce topological vortices in the $\langle d d\rangle$ phase: Abelian superfluid vortices, non-Abelian Alice strings, and doubly-wound non-Abelian strings. In section 3.4, we investigate generalized $\mathrm{AB}$ phases around vortices. In section 4 , we show topological vortex confinement mechanism in the confined phase. In section 5, for the consistency, we discuss the opposite ordering of symmetry breakings in which the 2SC condensate $\Phi_{2 \mathrm{SC}}$ develops VEVs first and $\Phi_{d d}$ condensate develops VEVs second. Section 6 is devoted to a summary and discussion. In appendix A, we introduce a pure color flux tube. In appendix B, we summarize the derivation of generalized $\mathrm{AB}$ phases.

\section{Two-flavor dense quark matter}

In this section, we set forth our setup in this paper by giving a short summary of the $2 \mathrm{SC}+\langle d d\rangle$ phase proposed in refs. [42, 43]. We also turn to the symmetry breaking induced 
by diquark condensation. The large part of analysis has already been carried out in the preceding work [77], however, here we introduce new idea of confined and deconfined phases of the vortices and classify them according to the value of the 2SC condensate. This notion will be important in the later discussions.

\section{$2.12 \mathrm{SC}+\langle d d\rangle$ phase}

Let us define the following diquark operators made out of up and down quark operators $\hat{u}$ and $\hat{d}$ :

$$
\left(\hat{\Phi}_{2 \mathrm{SC}}\right)^{\alpha} \equiv \epsilon^{\alpha \beta \gamma} \hat{u}_{\beta}^{T} \mathcal{C} \gamma^{5} \hat{d}_{\gamma}, \quad\left(\hat{\Phi}_{d d}\right)_{\alpha \beta}^{i j} \equiv \hat{d}_{\alpha}^{T} \mathcal{C} \gamma^{i} \nabla^{j} \hat{d}_{\beta}
$$

where $\mathcal{C}$ is the charge conjugation, Greek indices $(\alpha, \beta, \gamma, \ldots)$ are color indices of the fundamental representation, and Latin indices $(i, j, \ldots)$ denote spatial coordinates. In the latter diquark, the matrices $\gamma^{i}$ and spatial derivatives $\nabla^{j}$ correspond to spin and angular momentum in the ${ }^{3} P_{2}$ state, respectively. In the $2 \mathrm{SC}+\langle d d\rangle$ phase, these two diquark operators develop VEVs:

$$
\Phi_{2 \mathrm{SC}} \equiv\left\langle\hat{\Phi}_{2 \mathrm{SC}}\right\rangle, \quad \Phi_{d d} \equiv\left\langle\hat{\Phi}_{d d}\right\rangle
$$

Here, we make a distinction between condensates and operators, i.e., quantities without hats are condensates or VEVs and those with hats are operators. We assumed unitary gauge fixing for these expressions of diquark condensates. The conventional 2SC condensate is $\Phi_{2 \mathrm{SC}}$, and $\Phi_{d d}$, which is the ${ }^{3} P_{2}$ diquark pairing of $d$-quarks, is the new feature in the $2 \mathrm{SC}+\langle d d\rangle$ phase.

We note in passing that this $2 \mathrm{SC}+\langle d d\rangle$ phase was originally proposed in the context of quark-hadron continuity where neutron ${ }^{3} P_{2}$ superfluid and this $2 \mathrm{SC}+\langle d d\rangle$ phase are continuously connected $[42,43]$. The typical quark density range considered here is $0.1-1 \mathrm{fm}^{-3}$, which corresponds to several hundred $\mathrm{MeVs}$ of the quark chemical potential. The meaning of continuity becomes clear if one considers the order parameter operator of neutron ${ }^{3} P_{2}$ superfluid $[44,45,50]$ given by

$$
\hat{A}^{i j}=\hat{n}^{T} \mathcal{C} \gamma^{i} \nabla^{j} \hat{n}
$$

where $\hat{n}$ is a field operator of neutrons. The expectation value of $\hat{A}^{i j}$ in the hadronic phase reads

$$
\left\langle\hat{A}^{i j}\right\rangle=\left\langle\hat{n}^{T} \mathcal{C} \gamma^{i} \nabla^{j} \hat{n}\right\rangle
$$

It is finite as long as the ${ }^{3} P_{2}$ superfluidity of the neutron is realized. After rearranging the valence quark content, the expectation value of $\hat{A}^{i j}$ in the quark phase under the mean field approximation reads

$$
\left\langle\hat{A}^{i j}\right\rangle \simeq\left(\Phi_{2 \mathrm{SC}}\right)^{\alpha}\left(\Phi_{2 \mathrm{SC}}\right)^{\beta}\left(\Phi_{d d}\right)_{\alpha \beta}^{i j}
$$

The quantity $\left\langle\hat{A}^{i j}\right\rangle$ is also non-zero as long as $\Phi_{2 \mathrm{SC}}$ and $\Phi_{d d}$ are non-zero. As in the pure $2 \mathrm{SC}$ phase, $\Phi_{2 \mathrm{SC}}$ is non-vanishing. The $d d$ condensate $\Phi_{d d}$ is also non-vanishing owing 
to the coupling to the energy-momentum tensor. We consider the four-fermi coupling in the ${ }^{3} P_{2}$ diquark channel

$$
\hat{\mathcal{I}}=\left(\hat{\Phi}_{d d}\right)^{i j}\left(\hat{\Phi}_{d d}\right)_{i j} .
$$

By performing Fierz transformation, this operator $\hat{\mathcal{I}}$ has a direct correspondence in the fermionic energy-momentum tensor, $\hat{T}^{\mu \nu}=\overline{\hat{\gamma}} \psi i \gamma^{\mu} \partial^{\nu} \hat{\psi}$. The equilibrium value is $T^{\mu \nu}=$ $\operatorname{diag}[\varepsilon,-p,-p,-p]$, with the energy density $\varepsilon$ and the pressure $p$ of fermionic matter, and thus the expectation value of $\hat{\mathcal{I}}$ becomes

$$
\langle\hat{\mathcal{I}}\rangle \sim 3 p^{2} / 4
$$

which indicates the macroscopic expectation value of the ${ }^{3} P_{2}$ diquark interaction.

The VEV of the neutron superfluid operator is always non-zero in the hadronic and quark matter so the local order parameter cannot distinguish these two phases, leading to continuity.

\subsection{Symmetry of the $2 \mathrm{SC}+\langle d d\rangle$ phase: general consideration}

The relevant part of the symmetry of QCD in this work is $G_{\mathrm{QCD}}=\mathrm{SU}(3)_{\mathrm{C}} \times \mathrm{U}(1)_{\mathrm{B}}$. We will see in particular that $\mathrm{U}(1)_{\mathrm{B}}$ symmetry is broken so that topologically stable vortices can arise. An element $\left(U, e^{i \theta_{\mathrm{B}}}\right) \in G_{\mathrm{QCD}}$ acts on quark fields $\hat{q}$ as

$$
\hat{q} \rightarrow e^{i \theta_{\mathrm{B}}} \hat{q},
$$

where $U \in \mathrm{SU}(3)_{\mathrm{C}}$. The diquark condensates (2.2) transform as

$$
\Phi_{2 \mathrm{SC}} \rightarrow e^{2 i \theta_{\mathrm{B}}} U^{*} \Phi_{2 \mathrm{SC}}, \quad \Phi_{d d} \rightarrow e^{2 i \theta_{\mathrm{B}}} U \Phi_{d d} U^{T} .
$$

We assume an appropriate structure for the tensor indices $i, j$ of $\Phi_{d d},{ }^{1}$ and hereafter we suppress these indices for simplicity.

We discuss symmetry breaking patterns induced by the diquark condensations. Now we turn on each condensate sequentially instead of turning them on at the same time. Namely, the VEV of $\Phi_{d d}$ is firstly developed and then follows the VEV of $\Phi_{2 S C}$. The opposite ordering is also considered. It is summarized by the equations

$$
\begin{aligned}
& \text { 1. } G_{\mathrm{QCD}} \stackrel{\Phi_{d d}}{\longrightarrow} H_{d d} \stackrel{\Phi_{2 \mathrm{SC}}}{\longrightarrow} K_{2 \mathrm{SC}+d d} \\
& \text { 2. } G_{\mathrm{QCD}} \stackrel{\Phi_{2 \mathrm{SC}}}{\longrightarrow} \tilde{H}_{2 \mathrm{SC}} \stackrel{\Phi_{d d}}{\longrightarrow} K_{2 \mathrm{SC}+d d}
\end{aligned}
$$

and by schematic diagram in figure 1 . We begin with the first option (2.10) and then the second option (2.11) is mentioned to ensure the consistency that the physics does not depend on the ordering of the condensations. As shown below, in a certain assumption, there are at least two possibilities for $K_{2 \mathrm{SC}+d d}: K_{2 \mathrm{SC}+d d}^{\mathrm{deconf}}$ or $K_{2 \mathrm{SC}+d d}^{\mathrm{conf}}$.

\footnotetext{
${ }^{1}$ It is known in the nematic phase [49] for which $\operatorname{diag}(1, s, 1-s)$ is implied for the $i, j$ indices, with real parameter $s$.
} 


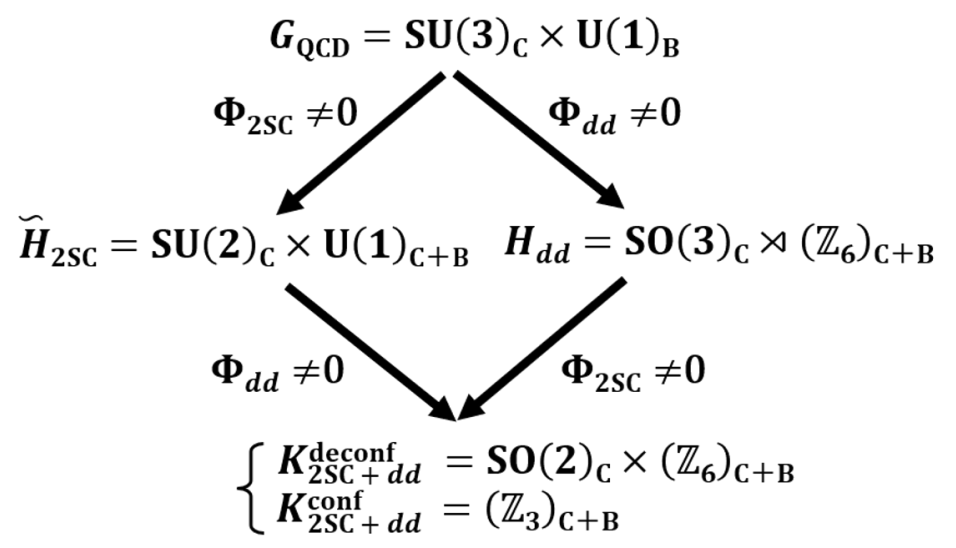

Figure 1. Spontaneous symmetry breaking patterns in the most symmetric case for $\Phi_{d d}$. $\Phi_{2 \mathrm{SC}}$ takes a value in $\mathbb{R}^{3}$ or $\mathbb{C}^{3}$ in the deconfined or confined phase where unbroken symmetry is $K_{2 \mathrm{SC}+d d}^{\text {deconf }}$ or $K_{2 \mathrm{SC}+d d}^{\mathrm{conf}}$, respectively.

\subsection{Symmetry breaking by the $\langle d d\rangle$ condensate}

Let us explain $G_{\mathrm{QCD}} \stackrel{\Phi_{d d}}{\longrightarrow} H_{d d}$ in eq. (2.10). By suitable gauge rotation, $\left(\Phi_{d d}\right)_{\alpha \beta}$ can be taken to be diagonal without loss of generality:

$$
\Phi_{d d}=\operatorname{diag}\left[\left(\Phi_{d d}\right)_{11},\left(\Phi_{d d}\right)_{22},\left(\Phi_{d d}\right)_{33}\right]
$$

We restrict ourselves to the simplest choice of $\Phi_{d d}$ :

$$
\Phi_{d d}=\Delta_{d d} \mathbf{1}_{3},
$$

i.e., each diagonal component of $\Phi_{d d}$ are taken to be equal: $\left(\Phi_{d d}\right)_{11}=\left(\Phi_{d d}\right)_{22}=\left(\Phi_{d d}\right)_{33}=$ $\Delta_{d d}$. The unbroken subgroup of $G_{\mathrm{QCD}}$ that keeps $\Phi_{d d}=\Delta_{d d} \mathbf{1}_{3}$ invariant is

$$
H_{d d}=\mathrm{SO}(3)_{\mathrm{C}} \rtimes\left(\mathbb{Z}_{6}\right)_{\mathrm{C}+\mathrm{B}} .
$$

This can be understood in the following sense: an element $\left(U, e^{i \theta_{\mathrm{B}}}\right) \in G_{\mathrm{QCD}}$ acts on $\Phi_{d d}=\Delta_{d d} \mathbf{1}_{3}$ as $\Delta_{d d} \mathbf{1}_{3} \rightarrow \Delta_{d d} e^{2 i \theta_{\mathrm{B}}} U U^{T}$, so that the condition under which the condensate does not change is $e^{2 i \theta_{\mathrm{B}}} U U^{T}=\mathbf{1}_{3}$. This condition is fulfilled by setting $U \in \mathrm{SO}(3)_{\mathrm{C}}$ and $e^{2 i \theta_{\mathrm{B}}}=1$. This accounts for $\mathrm{SO}(3)_{\mathrm{C}}$ in $H_{d d}$. The discrete group $\left(\mathbb{Z}_{6}\right)_{\mathrm{C}+\mathrm{B}}$ in $H_{d d}$ is defined by

$$
\left(\mathbb{Z}_{6}\right)_{\mathrm{C}+\mathrm{B}}:\left(X^{k}, \omega^{-2 k}\right) \in \mathrm{SU}(3)_{\mathrm{C}} \times \mathrm{U}(1)_{\mathrm{B}}=G_{\mathrm{QCD}},
$$

where $k=0,1,2,3,4,5$ and $\omega \equiv e^{i \pi / 3}$. Due to the semidirect product $\rtimes, X$ is subject to $\mathrm{SO}(3)_{\mathrm{C}}$ transformation and thus the expression is not unique (see ref. [77] for precise meaning of the semidirect product). The typical expressions of $X$ read

$$
X \equiv \operatorname{diag}\left(\omega, \omega, \omega^{-2}\right), \operatorname{diag}\left(\omega, \omega^{-2}, \omega\right), \operatorname{or} \operatorname{diag}\left(\omega^{-2}, \omega, \omega\right),
$$

generated by $\left(T_{8} \propto\right) \operatorname{diag}(1,1,-2), \operatorname{diag}(1,-2,1)$, or $\operatorname{diag}(-2,1,1)$, respectively of the broken $\mathrm{SU}(3)_{\mathrm{C}}$ symmetry. 
The order parameter manifold in this breaking $G_{\mathrm{QCD}} \rightarrow H_{d d}$ is

$$
\frac{G_{\mathrm{QCD}}}{H_{d d}}=\frac{\mathrm{SU}(3)_{\mathrm{C}} \times \mathrm{U}(1)_{\mathrm{B}}}{\mathrm{SO}(3)_{\mathrm{C}} \rtimes\left(\mathbb{Z}_{6}\right)_{\mathrm{C}+\mathrm{B}}} \simeq \frac{M_{3} \times S^{1}}{\left(\mathbb{Z}_{6}\right)_{\mathrm{C}+\mathrm{B}}}
$$

with $M_{3} \equiv \mathrm{SU}(3)_{\mathrm{C}} / \mathrm{SO}(3)_{\mathrm{C}}$. Because of the relation $\pi_{1}\left(G_{\mathrm{QCD}} / H_{d d}\right)=\mathbb{Z}$, non-Abelian Alice strings that appears in the later discussion can exist as topologically stable configuration. Higher homotopy groups are $\pi_{n}\left(G_{\mathrm{QCD}} / H_{d d}\right)=\pi_{n}\left(M_{3}\right)$ for $n>1$ and the latter can be found in refs. [89, 90]: $\pi_{2}\left(M_{3}\right)=\mathbb{Z}_{2}, \pi_{3}\left(M_{3}\right)=\mathbb{Z}_{4}$ and so on.

\subsection{Symmetry breaking by the 2SC condensate}

Next, we switch on the VEV of $\Phi_{2 \mathrm{SC}}$ in the existence of $\Phi_{d d}$. We explain $H_{d d} \stackrel{\Phi_{2 \mathrm{SC}}}{\longrightarrow} K_{2 \mathrm{SC}+d d}$ in eq. (2.10). The $\left(\mathbb{Z}_{6}\right)_{\mathrm{C}+\mathrm{B}}$ action on $\Phi_{2 \mathrm{SC}}$, as given in eq. (2.9), is

$$
\begin{aligned}
& \Phi_{2 \mathrm{SC}} \rightarrow \omega^{-2} X^{-1} \Phi_{2 \mathrm{SC}} \\
& =\left\{\begin{array}{l}
\omega^{-2} \operatorname{diag}\left(\omega, \omega, \omega^{-2}\right)^{-1} \Phi_{2 \mathrm{SC}}=\operatorname{diag}(-1,-1,1) \Phi_{2 \mathrm{SC}} \\
\omega^{-2} \operatorname{diag}\left(\omega, \omega^{-2}, \omega\right)^{-1} \Phi_{2 \mathrm{SC}}=\operatorname{diag}(-1,1,-1) \Phi_{2 \mathrm{SC}} \\
\omega^{-2} \operatorname{diag}\left(\omega^{-2}, \omega, \omega\right)^{-1} \Phi_{2 \mathrm{SC}}=\operatorname{diag}(1,-1,-1) \Phi_{2 \mathrm{SC} .} .
\end{array}\right.
\end{aligned}
$$

Then, we find that the unbroken symmetry depends on whether $\Phi_{2 \mathrm{SC}} \in \mathbb{R}^{3}$ or $\Phi_{2 \mathrm{SC}} \in \mathbb{C}^{3}$ :

$$
\begin{aligned}
& H_{d d} \rightarrow K_{2 \mathrm{SC}+d d} \\
& =\left\{\begin{array}{l}
K_{2 \mathrm{SC}+d d}^{\text {deconf }}=\mathrm{SO}(2)_{\mathrm{C}} \times\left(\mathbb{Z}_{6}\right)_{\mathrm{C}+\mathrm{B}} \text { for } \Phi_{2 \mathrm{SC}} \in \mathbb{R}^{3} \\
K_{2 \mathrm{SC}+d d}^{\mathrm{conf}}=\left(\mathbb{Z}_{3}\right)_{\mathrm{C}+\mathrm{B}} \text { for } \Phi_{2 \mathrm{SC}} \in \mathbb{C}^{3} .
\end{array}\right.
\end{aligned}
$$

The effects of this second symmetry breaking $H_{d d} \stackrel{\Phi_{2 \mathrm{SC}}}{\longrightarrow} K_{2 \mathrm{SC}+d d}$ on Alice strings are main topic of this and previous [77] papers. We focused on the deconfined phase in the case $\Phi_{2 \mathrm{SC}} \in \mathbb{R}^{3}$ in the preceding paper [77]; in this paper, we investigate the confined phase corresponding to the other possibility $\Phi_{2 \mathrm{SC}} \in \mathbb{C}^{3}$.

In the case of $\Phi_{2 \mathrm{SC}} \in \mathbb{R}^{3}$, the direction of $X \in \mathbb{Z}_{6}$ inside $G_{\mathrm{QCD}}$ is not unique but transforms under $\mathrm{SO}(3)_{\mathrm{C}}$. The three typical expressions of $X$ in eq. (2.16) keep

$$
\Phi_{2 \mathrm{SC}}^{T} \propto(0,0,1),(0,1,0),(1,0,0),
$$

unbroken, respectively. An $\mathrm{SO}(2)_{\text {C }}$ group remains intact, with a direct product with $X \in \mathbb{Z}_{6}$.

On the other hand, in the case of $\Phi_{2 S C} \in \mathbb{C}^{3}, \Phi_{2 S C}$ cannot be taken to be one component by using the $\mathrm{SO}(3)_{\mathrm{C}}$ symmetry, but at least two components should be nonvanishing. Consequently, only even numbers of the $\mathbb{Z}_{6}$ action in eq. (2.18), $\Phi_{2 \mathrm{SC}} \rightarrow \operatorname{diag}(1,1,1) \Phi_{2 \mathrm{SC}}$, remain unbroken, thus forming $\mathbb{Z}_{3}$.

The order parameter manifolds for these symmetry breakings are

$$
\begin{aligned}
\frac{H_{d d}}{K_{2 \mathrm{SC}+d d}^{\text {deconf }}} & =\frac{\mathrm{SO}(3)_{\mathrm{C}} \rtimes\left(\mathbb{Z}_{6}\right)_{\mathrm{C}+\mathrm{B}}}{\mathrm{SO}(2)_{\mathrm{C}} \times\left(\mathbb{Z}_{6}\right)_{\mathrm{C}+\mathrm{B}}} \simeq \frac{\mathrm{SO}(3)_{\mathrm{C}}}{\mathrm{SO}(2)_{\mathrm{C}}} \simeq S^{2}, \\
\frac{H_{d d}}{K_{2 \mathrm{SC}+d d}^{\text {conf }}} & =\frac{\mathrm{SO}(3)_{\mathrm{C}} \rtimes\left(\mathbb{Z}_{6}\right)_{\mathrm{C}+\mathrm{B}}}{\left(\mathbb{Z}_{3}\right)_{\mathrm{C}+\mathrm{B}}} .
\end{aligned}
$$




\subsection{The opposite ordering of symmetry breakings}

The opposite ordering of the condensation as in eq. (2.11) is discussed; we first consider the 2SC phase by $\Phi_{2 \mathrm{SC}}$ and subsequently consider $\Phi_{d d}$.

We choose the gauge of the 2SC condensate $\Phi_{2 \mathrm{SC}}$ as $\left(\Phi_{2 \mathrm{SC}}\right)^{\alpha}=\Delta_{2 \mathrm{SC}} \delta^{\alpha 3}$, and this spontaneously breaks $G_{\mathrm{QCD}}$ into

$$
\tilde{H}_{2 \mathrm{SC}}=\mathrm{SU}(2)_{\mathrm{C}} \times \mathrm{U}(1)_{\mathrm{C}+\mathrm{B}},
$$

where the $\mathrm{U}(1)_{\mathrm{C}+\mathrm{B}}$ symmetry is given by

$$
\left\{\left(e^{i \alpha T_{8}}, e^{2 i \alpha}\right) \in \mathrm{SU}(3)_{\mathrm{C}} \times \mathrm{U}(1)_{\mathrm{B}}: T_{8}=\operatorname{diag}(1,1,-2)\right\} .
$$

The order parameter manifold of this breaking is

$$
\frac{G_{\mathrm{QCD}}}{\tilde{H}_{2 \mathrm{SC}}}=\frac{\mathrm{SU}(3)_{\mathrm{C}}}{\mathrm{SU}(2)_{\mathrm{C}}},
$$

allowing trivial first homotopy group or no stable vortices as known before. Equivalently, this corresponds to the absence of superfluidity.

Let us switch on the $\Phi_{d d}$ condensate. The $\mathrm{SU}(2)_{\mathrm{C}}$ symmetry in $\tilde{H}_{2 \mathrm{SC}}$ diagonalizes upper-left block of $\Phi_{d d}$ :

$$
\Phi_{d d}=\left(\begin{array}{ccc}
\Delta_{d d}^{\prime} & 0 & \left(\Phi_{d d}\right)_{13} \\
0 & \Delta_{d d}^{\prime} & \left(\Phi_{d d}\right)_{23} \\
\left(\Phi_{d d}\right)_{13} & \left(\Phi_{d d}\right)_{23} & \Delta_{d d}^{\prime \prime}
\end{array}\right) .
$$

We assume here the diagonal components of the upper-left block to be equal. Unbroken symmetry is identified by looking at the $\mathbb{Z}_{6}$ action in eq. (2.15) with $X=\operatorname{diag}\left(\omega, \omega, \omega^{-2}\right)$ in eq. (2.16):

$$
\begin{aligned}
\Phi_{d d} & \rightarrow \omega^{-2} X \Phi_{d d} X^{T} \\
& =\left(\begin{array}{ccc}
\Delta_{d d}^{\prime} & 0 & \omega^{3}\left(\Phi_{d d}\right)_{13} \\
0 & \Delta_{d d}^{\prime} & \omega^{3}\left(\Phi_{d d}\right)_{23} \\
\omega^{3}\left(\Phi_{d d}\right)_{13} & \omega^{3}\left(\Phi_{d d}\right)_{23} & \Delta_{d d}^{\prime \prime}
\end{array}\right) .
\end{aligned}
$$

There are two phases, deconfined or confined, depending on if the off-diagonal entries are absent or not. In the previous paper [77], we investigated the case for the vanishing offdiagonal components. The corresponding unbroken group is $K=K_{2 \mathrm{SC}+d d}^{\mathrm{deconf}} \simeq \mathrm{SO}(2)_{\mathrm{C}} \times$ $\left(\mathbb{Z}_{6}\right)_{\mathrm{C}+\mathrm{B}}$ as the same as the first possibility of eq. (2.19). In this paper, our main focus is on the case that the off-diagonal components are present corresponding to the case $\Phi_{2 \mathrm{SC}} \in \mathbb{C}^{3}$ in eq. (2.10). In this case, applying the $\mathbb{Z}_{6}$ action even times in eq. (2.26) makes $\Phi_{d d}$ invariant because of $\omega^{6}=1$, thus confirming $K=K_{2 \mathrm{SC}+d d}^{\mathrm{conf}} \simeq\left(\mathbb{Z}_{3}\right)_{\mathrm{C}+\mathrm{B}}$ in the second possibility of eq. (2.19).

The order parameter manifolds for these breakings are

$$
\begin{aligned}
\frac{\tilde{H}_{2 \mathrm{SC}}}{K_{2 \mathrm{SC}+d d}^{\text {deconf }}} & =\frac{\mathrm{SU}(2)_{\mathrm{C}}}{\mathrm{SO}(2)_{\mathrm{C}}} \times \frac{\mathrm{U}(1)_{\mathrm{C}+\mathrm{B}}}{\left(\mathbb{Z}_{6}\right)_{\mathrm{C}+\mathrm{B}}} \simeq S^{2} \times \frac{\mathrm{U}(1)_{\mathrm{C}+\mathrm{B}}}{\left(\mathbb{Z}_{6}\right)_{\mathrm{C}+\mathrm{B}}}, \\
\frac{\tilde{H}_{2 \mathrm{SC}}}{K_{2 \mathrm{SC}+d d}^{\mathrm{conf}}} & =\mathrm{SU}(2)_{\mathrm{C}} \times \frac{\mathrm{U}(1)_{\mathrm{C}+\mathrm{B}}}{\left(\mathbb{Z}_{3}\right)_{\mathrm{C}+\mathrm{B}}} .
\end{aligned}
$$


In the both cases, the ground state admits topologically stable vortex configurations according to $\pi_{1}\left(\tilde{H}_{2 \mathrm{SC}} / K_{2 \mathrm{SC}+d d}^{\text {deconf }}\right) \simeq \mathbb{Z}$ and $\pi_{1}\left(\tilde{H}_{2 \mathrm{SC}} / K_{2 \mathrm{SC}+d d}^{\text {conf }}\right) \simeq \mathbb{Z}$. However, the difference in the discrete groups, $\left(\mathbb{Z}_{3}\right)_{\mathrm{C}+\mathrm{B}}$ or $\left(\mathbb{Z}_{6}\right)_{\mathrm{C}+\mathrm{B}}$, in the unbroken subgroups implies that minimal vortices are $1 / 6$ or $1 / 3$ winding in $\mathrm{U}(1)_{\mathrm{B}}$, thus carrying $1 / 6$ or $1 / 3$ circulations, respectively.

Comparing the order parameters for the two ways of the symmetry breakings, eqs. (2.10) and (2.11), look different at a first glance. However, these two are gauge equivalent and can be transformed to each other by a gauge transformation, implying the gauge invariant $\hat{A}^{i j}$ are the same.

\subsection{The overall symmetry breaking $G \rightarrow K$}

At this stage, we have the overall order parameter manifolds for the whole symmetry breakings

$$
\begin{aligned}
\frac{G}{K_{2 \mathrm{SC}+d d}^{\text {deconf }}} & =\frac{\mathrm{SU}(3)_{\mathrm{C}} \times \mathrm{U}(1)_{\mathrm{B}}}{\mathrm{SO}(2)_{\mathrm{C}} \times\left(\mathbb{Z}_{6}\right)_{\mathrm{C}+\mathrm{B}}} \simeq \frac{\mathrm{U}(3)}{\mathrm{SO}(2) \times \mathbb{Z}_{2}} \\
\frac{G}{K_{2 \mathrm{SC}+d d}^{\text {conf }}} & =\frac{\mathrm{SU}(3)_{\mathrm{C}} \times \mathrm{U}(1)_{\mathrm{B}}}{\left(\mathbb{Z}_{3}\right)_{\mathrm{C}+\mathrm{B}}} \simeq \mathrm{U}(3)
\end{aligned}
$$

for the deconfined and confined phases, respectively. Eventually, the order parameter manifold $\mathrm{U}(3)$ in eq. (2.28) for the confining phase is the same with that of the CFL phase [56].

\section{Topological vortices in $\langle d d\rangle$ phase}

In this section, we summarize vortices that appear in the presence of $\Phi_{d d}$. In section 3.1, we introduce an Abelian superfluid vortex. In section 3.2, we introduce a non-Abelian Alice vortex as the topologically most stable minimal configuration in the $\langle d d\rangle$ phase. In section 3.3, we also discuss doubly-wound non-Abelian string, which loses the most properties of the Alice strings. Generalized AB phases around these strings are summarized in section 3.4 .

\subsection{Abelian superfluid vortices}

The simplest vortex is an Abelian superfluid vortex:

$$
\Phi_{d d}(r, \varphi)=f_{0}(r) e^{i \varphi} \Delta_{d d} \mathbf{1}_{3} \sim e^{i \varphi} \Delta_{d d} \mathbf{1}_{3}
$$

where $(r, \varphi)$ is the polar coordinates. The boundary condition for the profile function $f_{0}$ is set as $f_{0}(0)=0$ and $f_{0}(\infty)=1$. The factor $e^{i \varphi}$ accounts for a unit quantized winding in $\mathrm{U}(1)_{\mathrm{B}}$. We call this a $\mathrm{U}(1)_{\mathrm{B}}$ superfluid vortex or an Abelian vortex. See the first line of table 1 . Due to the relation $\pi_{1}\left[\mathrm{U}(1)_{\mathrm{B}}\right]=\mathbb{Z}$, this string is topologically stable, however, it is unstable against decay into more stable vortices, i.e., triads of non-Abelian Alice strings with different color fluxes canceled as a whole. In the presence of $\Phi_{2 S C}$, this string remains unstable against decay as discussed in the previous paper [77]. However, as discussed in this paper, this becomes stable in the confined phase. 


\subsection{Non-Abelian Alice strings}

The most stable vortex is what we call as a non-Abelian Alice string. It is has orientational moduli in the internal gauge space of non-Abelian gauge group. The holonomy at infinite distance

$$
U(\varphi)=\mathcal{P} \exp \left(i \int_{0}^{\varphi} \boldsymbol{A} \cdot d \boldsymbol{\ell}\right)
$$

generates the condensate winding at spatial infinity

$$
\Phi_{d d}(\varphi)=e^{i \varphi / 3} U(\varphi) \Phi_{d d}(\varphi=0) U^{T}(\varphi),
$$

where $\Phi_{d d}(\varphi=0)=\Delta_{d d} \mathbf{1}_{3}$. The three representative configurations can be given by

$$
\begin{aligned}
\Phi_{d d}(r, \varphi) & =\Delta_{d d}\left(\begin{array}{ccc}
g(r) & 0 & 0 \\
0 & g(r) & 0 \\
0 & 0 & f(r) e^{i \varphi}
\end{array}\right), \\
U(\varphi) & =e^{i(\varphi / 6) \operatorname{diag}(-1,-1,2)}, \\
A_{i} & =-\frac{a(r)}{6 g} \frac{\epsilon_{i j} x^{j}}{r^{2}} \operatorname{diag}(-1,-1,2)
\end{aligned}
$$

for a blue (b) color magnetic flux

$$
\begin{aligned}
\Phi_{d d}(r, \varphi) & =\Delta_{d d}\left(\begin{array}{ccc}
g(r) & 0 & 0 \\
0 & f(r) e^{i \varphi} & 0 \\
0 & 0 & g(r)
\end{array}\right), \\
U(\varphi) & =e^{i(\varphi / 6) \operatorname{diag}(-1,2,-1)}, \\
A_{i} & =-\frac{a(r)}{6 g} \frac{\epsilon_{i j} x^{j}}{r^{2}} \operatorname{diag}(-1,2,-1)
\end{aligned}
$$

for a green $(g)$ color magnetic flux, and

$$
\begin{aligned}
\Phi_{d d}(r, \varphi) & =\Delta_{d d}\left(\begin{array}{ccc}
f(r) e^{i \varphi} & 0 & 0 \\
0 & g(r) & 0 \\
0 & 0 & g(r)
\end{array}\right), \\
U(\varphi) & =e^{i(\varphi / 6) \operatorname{diag}(2,-1,-1)}, \\
A_{i} & =-\frac{a(r)}{6 g} \frac{\epsilon_{i j} x^{j}}{r^{2}} \operatorname{diag}(2,-1,-1)
\end{aligned}
$$

for a red $(r)$ color magnetic flux. Here, $f$ and $g$ are the profile functions with the boundary conditions

$$
f(0)=g^{\prime}(0)=a(0)=0, \quad f(\infty)=g(\infty)=a(\infty)=1 .
$$

This carries $1 / 6$ quantized color-magnetic flux, $\mathcal{F}=\mathcal{F}_{0} / 6$ (see eq. (A.4) for the definition of $\mathcal{F}_{0}$ ) as well as $1 / 3$ quantized $\mathrm{U}(1)_{\mathrm{B}}$ circulation, as summarized in the second line of table 1. As $\Phi_{d d}$ have to be singlevalued, $U(2 \pi)$ belongs to the little group $H_{d d}$ of the condensate $\Phi_{d d}(0)$. Thus, this configuration connects two elements of $\operatorname{SU}(3)_{\mathrm{C}}: U(\varphi=0)=\mathbf{1}_{3}$ and 
$U(\varphi=2 \pi)=\operatorname{diag}\left(\omega^{-1}, \omega^{-1}, \omega^{2}\right),\left(\omega^{-1}, \omega^{2}, \omega^{-1}\right)$, or $\left(\omega^{2}, \omega^{-1}, \omega^{-1}\right)$ for the configuration in eq. (3.4), (3.5), or (3.6), respectively. All of these configurations with different color fluxes are continuously connected by Nambu-Goldstone (NG) modes associated with this spontaneous symmetry breaking in the vicinity of the vortex:

$$
O \in \frac{H_{d d}}{\tilde{K}_{\text {vortex }}}=\frac{\mathrm{SO}(3)_{\mathrm{C}} \rtimes \mathbb{Z}_{6}}{\mathrm{O}(2)_{\mathrm{C}} \times \mathbb{Z}_{6}} \simeq S^{2} / \mathbb{Z}_{2} \simeq \mathbb{R} P^{2} .
$$

In the vortex core, there remains the unbroken gauge symmetry.

One of the characteristic features of the Alice string is the presence of the so-called topological obstruction [19, 78-86, 91], stating that the unbroken generators $T_{x}, T_{y}$ and $T_{z}$ of $\mathrm{SO}(3)_{\mathrm{C}}$ are not globally defined around the string. These generators receive the transformation

$$
T_{x, y, z}(\varphi) \equiv U(\varphi) T_{x, y, z} U^{-1}(\varphi),
$$

around the string, and then we find

$$
\begin{aligned}
T_{y, z}(\varphi=2 \pi) & =-T_{y, z} \neq T_{y, z}(\varphi=0) \\
T_{x}(\varphi=2 \pi) & =+T_{x}=T_{x}(\varphi=0)
\end{aligned}
$$

for the Alice string with the flux of the color $r$ in eq. (3.4),

$$
\begin{gathered}
T_{z, x}(\varphi=2 \pi)=-T_{z, x} \neq T_{z, x}(\varphi=0) \\
T_{y}(\varphi=2 \pi)=+T_{y}=T_{y}(\varphi=0)
\end{gathered}
$$

for the one with the flux of the color $g$ in eq. (3.5), and

$$
\begin{aligned}
T_{x, y}(\varphi=2 \pi) & =-T_{x, y} \neq T_{x, y}(\varphi=0) \\
T_{z}(\varphi=2 \pi) & =+T_{z}=T_{z}(\varphi=0)
\end{aligned}
$$

for the one with the flux of the color $b$ in eq. (3.6). We can recover all the original $T_{x, y, z}$ by rotating $\varphi=4 \pi$ :

$$
T_{x, y, z}(\varphi=4 \pi)=T_{x, y, z}(\varphi=0),
$$

for any kind of Alice strings.

\subsection{Doubly-wound non-Abelian strings}

Among multiply-wound strings, a particularly important string is a doubly-wound nonAbelian string, given by

$$
\begin{aligned}
\Phi_{d d}(\varphi) & =e^{2 i \varphi / 3} U(\varphi) \Phi_{d d}(\varphi=0) U^{T}(\varphi) \\
& =\Delta_{d d}\left(\begin{array}{ccc}
g(r) & 0 & 0 \\
0 & g(r) & 0 \\
0 & 0 & f(r) e^{2 i \varphi}
\end{array}\right), \\
U(\varphi) & =e^{i(\varphi / 3) \operatorname{diag}(1,1,-2)} \\
A_{i} & =-\frac{a(r)}{3 g} \frac{\epsilon_{i j} x^{j}}{r^{2}} \operatorname{diag}(1,1,-2)
\end{aligned}
$$


for the color flux $b$, and similar for other color fluxes. This carries $1 / 3$ quantized color magnetic flux, $\mathcal{F}=\mathcal{F}_{0} / 3$, and $2 / 3$ quantized circulation. See the fifth line of table 1 . Since $U(\varphi=2 \pi)=e^{i(2 \pi / 3) \operatorname{diag}(-1,-1,2)}=e^{-2 \pi i / 3} \mathbf{1}_{3}$ this configuration connects two elements $\mathbf{1}_{3}$ and $\omega^{-2}$ of the center of $\mathrm{SU}(3)_{\mathrm{C}}{ }^{2}$ in contrast to a single non-Abelian Alice string which does not connect center elements of $\mathrm{SU}(3)_{\mathrm{C}}$. As we will see below, this string loses the most of Alice properties that a single Alice string possesses; there is no topological obstruction from eq. (3.13), and bears only color singlet $\mathrm{AB}$ phases as shown in the next section. Nevertheless, it has a color magnetic flux and the same moduli space with that in eq. (3.8) of a single Alice string.

In the $\langle d d\rangle$ phase, this string is unstable against a decay into two non-Abelian Alice strings of the same color because of superfluidity. In the presence of $\Phi_{2 S C}$, this remains unstable in the deconfined phase, but it will be stabilized in the confined phase, as discussed in later sections.

\subsection{Generalized Aharonov-Bohm phases}

Here we summarize AB phases of particles and condensation encircling the above introduced strings. In the CFL case, the $\mathrm{AB}$ phases in electromagnetic sector around a nonAbelian vortex was calculated [92], while those in color $\mathrm{SU}(3)_{\mathrm{C}}$ sector is $\mathbb{Z}_{3}$ [35]. In this section, we consider $\mathrm{AB}$ phases of color $\mathrm{SU}(3)_{\mathrm{C}}$ symmetry around a vortex, without switching on the electromagnetism.

First, we place the quark field $\hat{q}$, the gauge field $A_{i}$, and the 2SC diquark operator $\hat{\Phi}_{2 \mathrm{SC}}$ in the vortex configuration given above. When they wind around the vortex, they receive a gauge transformation according to the holonomy action in eq. (3.2), as well as a $\mathrm{U}(1)_{\mathrm{B}}$ transformation if it participates in the condensation with a vortex winding. Thus, after $2 \pi$ winding around the vortex, the fields receive a phase from the Wilson loop and the $\mathrm{U}(1)_{\mathrm{B}}$ baryon circulation. The phase without the baryon circulation is called as (pure) AB phase while the one with the baryon circulation as generalized AB phase. The former is relevant for heavy quarks such as strange quarks $s$ that do not participate in the condensation, while the latter is necessary for light quarks $u$ and $d$, since they participate in condensations $\Phi_{d d}$ containing a vortex configuration. In the later sections, a criterion for the existence of the confinement will be given by this (generalized) AB phase.

At any azimuthal angle $\varphi \neq 0$, the light quark field operator $\hat{q}$ and the diquark operator $\hat{\Phi}_{2 \mathrm{SC}}$ are expressed by a holonomy action as

$$
\begin{aligned}
\hat{q}(\varphi) & \sim e^{i \theta_{\mathrm{B}}(\varphi)} U(\varphi) \hat{q}(\varphi=0), \\
\hat{\Phi}_{2 \mathrm{SC}}(\varphi) & \sim e^{2 i \theta_{\mathrm{B}}(\varphi)} U^{-1}(\varphi) \hat{\Phi}_{2 \mathrm{SC}}(\varphi=0),
\end{aligned}
$$

respectively, where $U(\varphi)$ is defined as in eq. (3.2). As mentioned above, the light quarks $u$ and $d$ that participate in the condensations, and $\hat{\Phi}_{2 S C}$ receive an additional contributionfrom the baryon number symmetry $\mathrm{U}(1)_{\mathrm{B}}$ other than the usual $\mathrm{AB}$ phase of the color

\footnotetext{
${ }^{2}$ It is also worth to point out that this property connecting two center elements of $\mathrm{SU}(3)_{\mathrm{C}}$ is shared by non-Abelian strings in the CFL phase [56, 59].
} 


\begin{tabular}{|c|c|c|c|c|c|c|c|c|c|c|}
\hline phase & vortex & $\begin{array}{c}\mathrm{U}(1)_{\mathrm{B}} \\
\text { circul. } B\end{array}$ & $\begin{array}{c}\text { color } \\
\text { magnetic } \\
\text { flux } \mathcal{F} \\
\end{array}$ & $\begin{array}{l}\text { generalized } \\
\text { AB phase for } \\
(u, d) \text { quarks }\end{array}$ & & $\begin{array}{l}\text { AB phase for } \\
s \text { quark }\end{array}$ & & $\begin{array}{c}\text { generalized } \\
\text { AB phase for } \\
\hat{\Phi}_{2 \mathrm{SC}}\end{array}$ & & $\begin{array}{l}\text { color } \\
\text { reps. }\end{array}$ \\
\hline (de)conf & $\mathrm{U}(1)_{\mathrm{B}}$ vortex & 1 & 0 & $(-1,-1,-1)$ & $\mathbb{Z}_{2}$ & $(1,1,1)$ & 1 & $(1,1,1)$ & 1 & singlet \\
\hline deconf & $\begin{array}{c}\mathrm{U}(1)_{\mathrm{C}}(\mathrm{d}) \text { or } \\
\text { pure color flux }(\mathrm{d}) \\
\mathrm{U}(1)_{\mathrm{C}}(\mathrm{c}) \text { or } \\
\text { pure color flux }(\mathrm{c})\end{array}$ & 0 & $1 / 2$ & $\begin{array}{l}\left(\begin{array}{lll}+1 & -1 & -1 \\
-1 & +1 & -1 \\
-1 & -1 & +1\end{array}\right) \\
\left(\begin{array}{lll}+1 & +1 & +1 \\
+1 & +1 & +1 \\
+1 & +1 & +1\end{array}\right)\end{array}$ & $\mathbb{Z}_{2}$ & $\begin{array}{l}\left(\begin{array}{lll}+1 & -1 & -1 \\
-1 & +1 & -1 \\
-1 & -1 & +1\end{array}\right) \\
\left(\begin{array}{lll}+1 & +1 & +1 \\
+1 & +1 & +1 \\
+1 & +1 & +1\end{array}\right)\end{array}$ & $\mathbb{Z}_{2}$ & $\begin{array}{l}\left(\begin{array}{lll}+1 & -1 & -1 \\
-1 & +1 & -1 \\
-1 & -1 & +1\end{array}\right) \\
\left(\begin{array}{lll}+1 & +1 & +1 \\
+1 & +1 & +1 \\
+1 & +1 & +1\end{array}\right)\end{array}$ & $\mathbb{Z}_{2}$ & non-singlet \\
\hline deconf & $\begin{array}{l}\text { NA Alice string } \\
\text { or } \mathrm{U}(1)_{\mathrm{C}+\mathrm{B}}(\mathrm{d}) \\
\text { doubly-wound } \\
\mathrm{NA} \text { string } \\
\text { or } \mathrm{U}(1)_{\mathrm{C}+\mathrm{B}}(\mathrm{c})\end{array}$ & $1 / 3$ & $1 / 6$ & $\begin{array}{l}\left(\begin{array}{lll}-1 & +1 & +1 \\
+1 & -1 & +1 \\
+1 & +1 & -1\end{array}\right) \\
\left(\begin{array}{lll}+1 & +1 & +1 \\
+1 & +1 & +1 \\
+1 & +1 & +1\end{array}\right)\end{array}$ & $\mathbb{Z}_{2}$ & $\begin{array}{c}\left(\begin{array}{ccc}\omega^{2} & \omega^{-1} & \omega^{-1} \\
\omega^{-1} & \omega^{2} & \omega^{-1} \\
\omega^{-1} & \omega^{-1} & \omega^{2}\end{array}\right) \\
\left(\begin{array}{llll}\omega^{4} & \omega^{4} & \omega^{4} \\
\omega^{4} & \omega^{4} & \omega^{4} \\
\omega^{4} & \omega^{4} & \omega^{4}\end{array}\right)\end{array}$ & $\mathbb{Z}_{6}$ & $\begin{array}{l}\left(\begin{array}{lll}+1 & -1 & -1 \\
-1 & +1 & -1 \\
-1 & -1 & +1\end{array}\right) \\
\left(\begin{array}{lll}+1 & +1 & +1 \\
+1 & +1 & +1 \\
+1 & +1 & +1\end{array}\right)\end{array}$ & $\mathbb{Z}_{2}$ & non-singlet \\
\hline
\end{tabular}

Table 1. (Generalized) AB phases of light $(u, d)$ quarks, heavy $(s)$ quark, and the $2 \mathrm{SC}$ condensate $\Phi_{2 \mathrm{SC}} \sim u d$ around various vortices introduced in this section (a pure color flux is introduced in appendix A). For row vectors, their columns represent the colors of the quarks or $\Phi_{2 \mathrm{SC}}$ encircling the vortex. For $3 \times 3$ matrices, rows represent the colors of fluxes of the vortices and columns represent the colors of the quarks or $\Phi_{2 \mathrm{SC}}$. The order $k$ of the $s$-quark AB phase $\mathbb{Z}_{k}$ corresponds to the flux $1 / k$ of the vortex.

gauge group, because $\hat{\Phi}_{2 \mathrm{SC}}$ itself contains a vortex winding. The total phase is a generalized AB phase. The generalized $\mathrm{AB}$ phase $\Gamma$ in the exponentiated form can be read out from the fields at $\varphi=2 \pi$ after going around the vortex, i.e., $\hat{q}(0) \rightarrow \hat{q}(2 \pi)=\Gamma \hat{q}(0)$ and $\hat{\Phi}_{2 \mathrm{SC}}(0) \rightarrow \hat{\Phi}_{2 \mathrm{SC}}(2 \pi)=\Gamma \hat{\Phi}_{2 \mathrm{SC}}(0)$. The gauge field $A_{i}$ in the vortex configuration is proportional to the diagonal matrix, so taking $A_{i} \propto \operatorname{diag}(-1,-1,2)$ for instance for the color flux $b$, the explicit form of the field is

$$
\begin{aligned}
\hat{q}(2 \pi) & \sim e^{i \theta_{\mathrm{B}}(\varphi)} U(2 \pi) \hat{q}(0) \\
& \sim e^{i \pi B} e^{2 i \pi \mathcal{F} \operatorname{diag}(-1,-1,2)}\left(\begin{array}{c}
\hat{q}_{r}(0) \\
\hat{q}_{g}(0) \\
\hat{q}_{b}(0)
\end{array}\right), \\
\hat{\Phi}_{2 \mathrm{SC}}(2 \pi) & \sim e^{2 i \theta_{\mathrm{B}}(\varphi)} U^{-1}(2 \pi) \hat{\Phi}_{2 \mathrm{SC}}(0) \\
& \sim e^{2 i \pi B} e^{-2 i \pi \mathcal{F} \operatorname{diag}(-1,-1,2)}\left(\begin{array}{c}
\hat{\Phi}_{2 \mathrm{SC}}^{r}(0) \\
\hat{\Phi}_{2 \mathrm{SC}}^{g}(0) \\
\hat{\Phi}_{2 \mathrm{SC}}^{b}(0)
\end{array}\right)
\end{aligned}
$$

where $B$ and $\mathcal{F}$ are the $\mathrm{U}(1)_{\mathrm{B}}$ circulation and the color-magnetic flux, respectively. We tabulate the values of $B$ and $\mathcal{F}$ for each kind of vortex in table 1 , and the detailed derivations of generalized $\mathrm{AB}$ phases are summarized in appendix B. When an $s$-quark that does not participate in the condensations encircles a vortex, it receives only an $\mathrm{AB}$ phase of the color gauge group as mentioned above. 


\section{Vortex confinement}

Now let us turn on the VEV of the $2 \mathrm{SC}$ condensate $\Phi_{2 \mathrm{SC}}$. In the previous paper, we considered the deconfined phase in which the 2SC condensates $\Phi_{2 \mathrm{SC}}$ are real-valued and can be taken to be one component by the $\mathrm{SO}(3)_{\mathrm{C}}$ gauge symmetry unbroken in the presence of the $\langle d d\rangle$ condensate. Here, we consider the confined phase where $\Phi_{2 S C}$ are generically complex-valued. In this case, one cannot take a gauge in which the $2 \mathrm{SC}$ condensate $\Phi_{2 \mathrm{SC}}$ develops a VEV only in one component, unlike the case of the deconfined phase. Instead, the $\Phi_{2 \mathrm{SC}}$ has at least two components as VEVs.

In section 4.1, we introduce $\mathrm{AB}$ defects attached to non-Abelian Alice strings. In section 4.2 we construct a baryonic molecule of three Alice strings connected by a domain wall junction, while in section 4.3 we construct a mesonic molecule of two Alice strings connected by a single domain wall. In section 4.4 we give a comment on a collision of two $\mathrm{U}(1)_{\mathrm{B}}$ strings decaying into three doubly-wound non-Abelian strings.

\subsection{Aharonov-Bohm defects in 2SC condensate $\Phi_{2 \mathrm{SC}}$}

Here, we consider all three components for generality. Then, when the 2SC condensate $\Phi_{2 \mathrm{SC}}$ encircles a single non-Abelian Alice string in eqs. (3.4), (3.5) and (3.6), it receives non-trivial AB phases summarized in eq. (B.20). More explicitly, it is

$$
\begin{aligned}
& \Phi_{2 \mathrm{SC}}^{\alpha}=\left(\begin{array}{c}
\Delta_{1} \\
\Delta_{2} \\
\Delta_{3}
\end{array}\right) \stackrel{\text { holonomy }}{\longrightarrow} \\
& \left\{\begin{array}{lll}
\left(\begin{array}{l}
-\Delta_{1} \\
-\Delta_{2} \\
+\Delta_{3}
\end{array}\right) & \text { around } M \sim\left(\begin{array}{lll}
1 & & \\
& 1 & \\
& & e^{i \varphi}
\end{array}\right) & (b) \\
\left(\begin{array}{l}
-\Delta_{1} \\
+\Delta_{2} \\
-\Delta_{3}
\end{array}\right) & \text { around } M \sim\left(\begin{array}{lll}
1 & & \\
& e^{i \varphi} & \\
& & 1
\end{array}\right) & (g) \\
\left(\begin{array}{l}
+\Delta_{1} \\
-\Delta_{2} \\
-\Delta_{3}
\end{array}\right) & \text { around } M \sim\left(\begin{array}{lll}
e^{i \varphi} & & \\
& 1 & \\
& & 1
\end{array}\right) & (r)
\end{array} .\right.
\end{aligned}
$$

Apparently, the inconsistency arises from non-singlevaluedness around the string if all $\Delta$ 's have VEVs. This could be avoided if only one of $\Delta$ 's has a VEV, corresponding to the deconfined phase leading to the bulk-soliton moduli locking. It is, however, unavoidable in the confined phase in which at least two of $\Delta$ 's must have VEVs.

To overcome this problem, we insert the following function $h(\varphi)$ in the $2 \mathrm{SC}$ condensate $\Phi_{2 S C}$ to maintain the singlevaluedness of $\Phi_{2 S C}$ :

$$
\Phi_{2 \mathrm{SC}}^{\alpha}= \begin{cases}\left(\begin{array}{c}
\Delta_{1} h(\varphi) \\
\Delta_{2} h(\varphi) \\
\Delta_{3}
\end{array}\right) & \text { for }(b) \\
\left(\begin{array}{c}
\Delta_{1} h(\varphi) \\
\Delta_{2} \\
\Delta_{3} h(\varphi)
\end{array}\right) & \text { for }(g) \\
\left(\begin{array}{c}
\Delta_{1} \\
\Delta_{2} h(\varphi) \\
\Delta_{3} h(\varphi)
\end{array}\right) & \text { for }(r)\end{cases}
$$




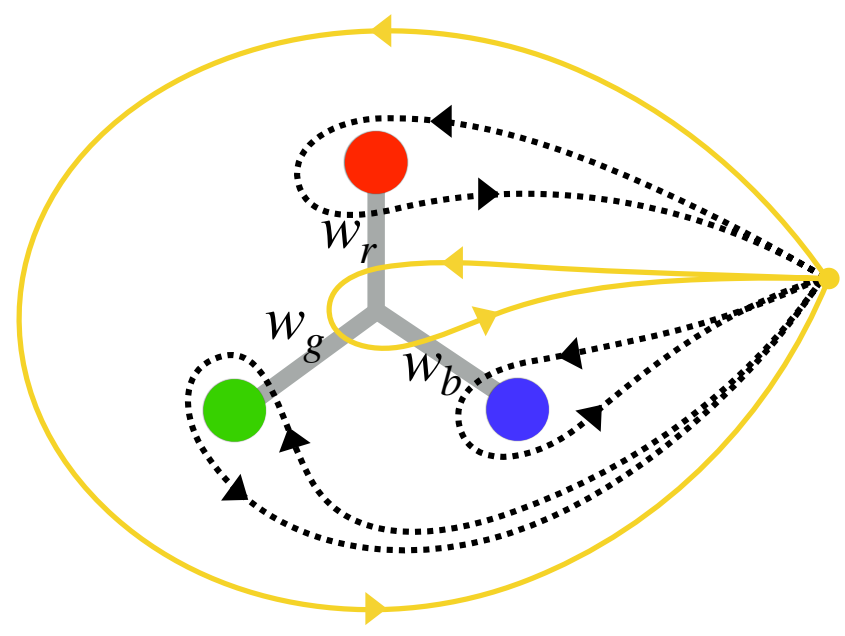

Figure 2. Schematic figure of a vortex baryon. In this case, three Alice strings with different color magnetic fluxes with the total color flux canceled out are confined by a domain wall junction denoted by grey lines. The black dotted loops encircling one of the three Alice strings show the Wilson-loops that pick up color non-singlet generalized $\mathrm{AB}$ phases, while the yellow loops show the color singlet ones. The large yellow loop encircles all of the three Alice strings and the small yellow loop encircles none of them but passes through the three domain walls.

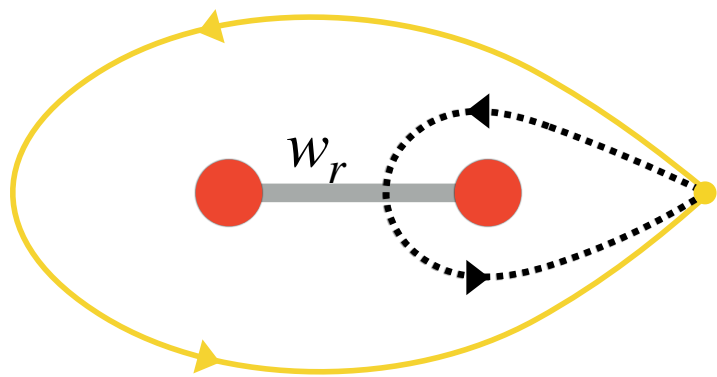

Figure 3. Schematic figure of a vortex meson. In this case, two Alice strings with the same color fluxes are confined and connected by a single domain wall. The black dotted loop encircling one of the two Alice strings shows the Wilson-loops that pick up color non-singlet generalized AB phase, while the yellow loop encircling the two Alice strings show the color singlet ones. Although the composite state carries a net color magnetic flux, it is a color singlet state in terms of the generalized $\mathrm{AB}$ phase.

for the three cases in eq. (4.1). Here $h(\varphi)$ is a kink profile inserted to compensate the AB phase, satisfying the boundary conditions $h(\varphi=0)=1$ and $h(\varphi=2 \pi)=-1$. The domain walls or solitons appearing to compensate an $\mathrm{AB}$ phase are called as the AB defects [19-21].

Alice strings are confined due to the formation of the AB defects. A single Alice string is attached to a single $\mathrm{AB}$ defect extending to infinity. For finite energy configurations, as we illustrate in figures 2 and 3, the $\mathrm{AB}$ defects are attached to the Alice strings so that they are confined. There are two kinds of confinements: "baryonic type" made of three Alice strings in figure 2 and "mesonic type" made of two Alice strings in figure 3, which we will discuss in the following subsections. 


\subsection{Baryonic molecule}

Let us discuss a molecule of the baryonic type. The three circles red, green and blue in figure 2 denote the vortices, and the lines attached to them are the kinks that we have considered above. We denote the kinks which are attached to the red, green and blue vortices as $w_{r}, w_{g}$ and $w_{b}$, respectively. We can show that these three kinks are put together at the center with the domain wall junction as follows. From eq. (4.2), we can define the domain wall operators

$$
\begin{aligned}
& w_{r}=\operatorname{diag}(-1,-1,1), \\
& w_{g}=\operatorname{diag}(-1,1,-1), \\
& w_{b}=\operatorname{diag}(1,-1,-1)
\end{aligned}
$$

that act on $\Phi_{2 \mathrm{SC}}$ : when the $\Phi_{2 \mathrm{SC}}$ passes through a domain wall, say $w_{r}$, it undergoes the transformation $w_{r} \Phi_{2 \mathrm{SC}}$. Then, we have a relation

$$
w_{r} w_{g} w_{b}=1
$$

implying that when two domain walls $w_{r}, w_{g}$ collide, it becomes $w_{b}^{-1}=w_{b}$. This implies that the three domain walls meet at a junction. In other words, along the small yellow loop in figure $2, \Phi_{2 \mathrm{SC}}$ passes through the all three domain walls, coming back to the original configuration due to eq. (4.4).

Dynamically, these three Alice strings are pulled by the domain walls because of their tensions. The fate of this baryonic configuration is nothing but a $\mathrm{U}(1)_{\mathrm{B}}$ superfluid vortex, having no color magnetic flux. In fact, color magnetic fluxes of $r, g, b$ are canceled out when they are combined together.

Our confinement argument relies on the generalized AB phases of the 2SC condensate $\Phi_{2 \mathrm{SC}}$ encircling the vortices, as we discussed above. Since $\Phi_{2 \mathrm{SC}}$ is color non-singlet carrying a color index, this leads us to a conjecture that the criterion of the confinement is that the (generalized) $\mathrm{AB}$ phases picking up the color of vortices should disappear at the spatial infinity in the confined phase. To check the validity of this conjecture, let us discuss generalized $\mathrm{AB}$ phases of $\Phi_{2 S C}$, light $u, d$-quarks, and a heavy $s$-quark.

Generalized Aharonov-Bohm phases of $\boldsymbol{\Phi}_{2 \text { SC }}$. If we look at the outer yellow path in figure 2, then its generalized $\mathrm{AB}$ phase is apparently trivial. However, if we go around each colored Alice strings, then we pick up a non-trivial generalized AB phase. These nontrivial $\mathrm{AB}$ phases are compensated by each kinks $w_{r}, w_{b}$, or $w_{g}$. Along the inner yellow path encircling the junction point, the $\Phi_{2 \mathrm{SC}}$ receives no $\mathrm{AB}$ phase but it receives domain wall operations in eq. (4.3) when it passes across a domain wall. However, it comes back to the original form due to the relation in eq. (4.4) at the end of the whole path.

Generalized Aharonov-Bohm phases of the light $\boldsymbol{u}, \boldsymbol{d}$-quarks. When a $u$ or $d$ quark encircles each Alice string, it picks up a nontrivial generalized AB phase only when it encircles an Alice string with the corresponding color, as can be seen from eq. (B.18), or the fourth line of table 1 . For instance, only $u_{r}\left(d_{r}\right)$ recives -1 when it encircles a red 
Alice string along the corresponding dotted loop in figure 2 , but $u_{g}\left(d_{g}\right)$ or $u_{b}\left(d_{b}\right)$ does not. Thus, the up or down quark can detect the color of the Alice string at the infinite distance. However, if each quark encircles all of the three Alice strings along the outer yellow loop in figure 2, it always picks up a generalized $\mathrm{AB}$ phase -1 irrespective of its color:

$$
\operatorname{diag}(1,1,-1) \times \operatorname{diag}(1,-1,1) \times \operatorname{diag}(-1,1,1)=\operatorname{diag}(-1,-1,-1)
$$

which is a color singlet. This phase does not have to be canceled because it develops no VEV, unlike the case of $\Phi_{2 S C}$. The right hand side coincides with the generalized AB phase of the $u, d$ quarks around a $\mathrm{U}(1)_{\mathrm{B}}$ vortex which is in fact a color singlet, see the first line of table 1. Thus, the $u, d$ quarks cannot detect the color of the baryonic molecule. This can be understood as the confinement.

Aharonov-Bohm phases of the heavy $s$-quark. Let us discuss a strange quark $s$ encircling the baryonic molecule or each Alice string. When it encircles one of Alice strings, say the red Alice string, $s_{r}$ receives $w^{2}$ while $s_{g}$ and $s_{b}$ receive $w^{-1}$, as can be seen from eq. (B.16) or the fourth line of table 1. Thus, the strange quark can detect the color of the Alice string at the infinite distance. However, when it encircles the baryonic molecule, neither $s_{r}, s_{g}$ nor $s_{b}$ receives any $\mathrm{AB}$ phase:

$$
\operatorname{diag}\left(\omega^{-1}, \omega^{-1}, \omega^{2}\right) \times \operatorname{diag}\left(\omega^{-1}, \omega^{2}, \omega^{-1}\right) \times \operatorname{diag}\left(\omega^{2}, \omega^{-1}, \omega^{-1}\right)=\operatorname{diag}(1,1,1)
$$

which is a color singlet. Again, the right hand side coincides with the AB phases of strange quarks around a $\mathrm{U}(1)_{\mathrm{B}}$ Abelian vortex, which is a color singlet, see the first line of table 1. This is also a consequence of the confinement.

In summary, we observe that each Alice string has a color non-singlet generalized $\mathrm{AB}$ phase and three Alice strings have a color singlet generalized $\mathrm{AB}$ phase as a whole it implies confinement. The $2 \mathrm{SC}$ condensate $\Phi_{2 \mathrm{SC}}$ receives color non-singlet generalized $\mathrm{AB}$ phases, so the $\mathrm{AB}$ defects should be generated to compensate the $\mathrm{AB}$ phases to ensure the singlevaluedness of $\Phi_{2 S C}$. Thus, each Alice string has to be confined by the AB defect. A baryonic configuration, made of three Alice strings of different color magnetic fluxes with the total color canceled out, has no generalized $\mathrm{AB}$ phase, where three Alice strings are connected by the domain wall junction. The domain wall tension pulls the three Alice strings to combine them, resulting in the $\mathrm{U}(1)_{\mathrm{B}}$ Abelian superfluid vortex.

\subsection{Mesonic molecule}

There is also another way of confinement. Two Alice strings with the same color fluxes are confined together as shown in figure 3. In this case, we do not have to introduce the domain wall junction, but simply two colored vortices are connected with the kink $w_{r}$. Dynamically, the two constituent Alice strings in the mesonic molecule are pulled by the domain wall tension, and they are combined together. The fate of this configuration is a doubly-wound non-Abelian string introduced in section 3.3, which in fact does not have the Alice properties.

One may wonder why the mesonic-type molecule having a color magnetic flux is allowed in the confined phase. This can be understood from the fact that no colored quarks can 
pick up an $\mathrm{AB}$ phase depending on color when it encircles the molecule along the outer yellow loop in figure 3, as explained below.

Generalized Aharonov-Bohm phases of $\mathbf{\Phi}_{\mathbf{2}} \mathbf{S C}$. If we look at the outer yellow path in figure 3, then its generalized $\mathrm{AB}$ phase is apparently trivial. However, if we go around each colored string, then we pick up a non-trivial generalized AB phase, which must be compensated by a kink $w_{r}$.

Generalized Aharonov-Bohm phases of the light $\boldsymbol{u}, \boldsymbol{d}$-quarks. A up or down quark receives no generalized $\mathrm{AB}$ phase when it encircles the molecule, while it receives a nontrivial generalized $\mathrm{AB}$ phase depending on its color, when it encircles each Alice string, as in eq. (B.18) or the fourth line of table 1 ; for instance only $u_{r}\left(d_{r}\right)$ recives -1 when it encircles a red Alice string, but $u_{g}\left(d_{g}\right)$ or $u_{b}\left(d_{b}\right)$ does not. This implies that the up or down quark can detect the color of the Alice string at the infinite distance but cannot do the color of the molecule;

$$
\operatorname{diag}(1,1,-1) \times \operatorname{diag}(1,1,-1)=\operatorname{diag}(1,1,1)
$$

The right hand side coincides with the generalized $\mathrm{AB}$ phases of $u, d$ quarks around a doubly-wound non-Abelian string, which is a color singlet, see eq. (B.24) or the fifth line of table 1. Thus, the all $u, d$ quarks receive no generalized $\mathrm{AB}$ phase irrespective of their color, thereby implying that the $u, d$ quarks cannot detect the color of the molecule.

Aharonov-Bohm phases of the heavy $s$-quark. It is further interesting to see what happens when a strange quark $s$ encircles the molecule or each Alice string. When it encircles one of red Alice strings, $s_{r}$ receives $w^{2}$ while $s_{g}$ and $s_{b}$ receive $w^{-1}$, and so the strange quark can detect the color of the Alice string at the infinite distance. However, when it encircles the molecule, $s_{r}$ receives $w^{4}$ and $s_{g}$ and $s_{b}$ receive $w^{-2}=w^{4}$. Therefore, we have the relation

$$
\operatorname{diag}\left(\omega^{-1}, \omega^{-1}, \omega^{2}\right) \times \operatorname{diag}\left(\omega^{-1}, \omega^{-1}, \omega^{2}\right)=\operatorname{diag}\left(\omega^{4}, \omega^{4}, \omega^{4}\right)
$$

which is nontrivial but a color singlet. The right hand side coincides with the $\mathrm{AB}$ phases of the $s$-quark around a doubly-wound non-Abelian string, which is a color singlet, see eq. (B.22) or the fifth line of table 1. Thus, all strange quarks receive the same AB phase irrespective of their color, thereby implying that the strange quark cannot detect the color of the molecule.

The notion of confinement should be used for color which can be detected by the (generalized) $\mathrm{AB}$ phases at infinity, but not for colors of fluxes that vortices have. The mesonic molecule possess colored magnetic flux, but it is not read out by the generalized $\mathrm{AB}$ phases as we showed above, so the mesonic molecule can be stated as confined.

It is interesting to point out that in this definition, non-Abelian vortices in the CFL phase are already confined as they are [70, 71]. 


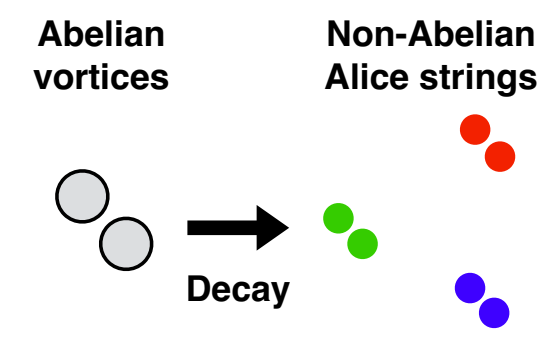

Figure 4. Decay of a doubly-wound $\mathrm{U}(1)_{\mathrm{B}}$ string into three doubly-wound non-Abelian strings with different color fluxes with the total color flux canceled out.

\subsection{Baryons-to-mesons decay}

Before closing this section, one comment is in order. In the confined phase, a $U(1)_{B}$ vortex cannot decay into three non-Abelian Alice strings because of the $\mathrm{AB}$ defects connecting them, as seen in the last subsection. However, if we prepare two $U(1)_{B}$ vortices at the same position, namely a doubly-wound $\mathrm{U}(1)_{\mathrm{B}}$ vortex, it can decay into three doublywound non-Abelian strings, as schematically drawn in figure 4. Of course, two $\mathrm{U}(1)_{\mathrm{B}}$ vortices themselves repel each other, and so it is not easy to prepare a doubly-wound $\mathrm{U}(1)_{\mathrm{B}}$ vortex. Also, once we prepare it, it is an open question which decay channel is more dominant between the decay into two $\mathrm{U}(1)_{\mathrm{B}}$ vortices or the decay into three doubly-wound non-Abelian strings.

\section{Consistency with the opposite ordering in the symmetry breaking}

In this section, we discuss vortices formed in the opposite ordering of the condensation given in eq. (2.11): the $2 \mathrm{SC}$ condensate $\Phi_{2 \mathrm{SC}}$ develops first, then the $\langle d d\rangle$ condensate. First, the 2SC condensate in the vacuum can be taken as $\left(\Phi_{2 \mathrm{SC}}\right)^{\alpha}=\Delta_{2 \mathrm{SC}} \delta^{\alpha 3}$ as usual for the 2SC phase. Second, vortices arise for the $\Phi_{d d}$ condensation due to $\pi_{1}\left(\tilde{H}_{2 \mathrm{SC}} / K_{2 \mathrm{SC}+d d}\right)=\mathbb{Z}$ with the order parameter manifolds in eq. (2.27), as already given in section 2.5.

\subsection{Superfluid vortex}

The configuration of a superfluid vortex is

$$
\begin{aligned}
\Phi_{d d}(\varphi) & =f_{0}(r) e^{i \varphi} \Delta_{d d} \mathbf{1}_{3}, \\
\Phi_{2 \mathrm{SC}}(\varphi) & =h_{0}(r) e^{i \varphi}\left(\begin{array}{lll}
0 & 0 & \Delta_{2 S C}
\end{array}\right)^{T}
\end{aligned}
$$

where we set the boundary conditions as

$$
f_{0}(0)=h_{0}(0)=0, \quad f_{0}(\infty)=h_{0}(\infty)=1 .
$$

This is supported by the breaking of the $\mathrm{U}(1)_{B}$ symmetry, and is exactly a $\mathrm{U}(1)_{B}$ vortex considered in section 3.1. This is nothing but a baryonic bound state of three Alice strings. 


\section{$5.2 \quad \mathrm{U}(1)_{\mathrm{C}+\mathrm{B}}$ vortices}

Here, we consider the vortex with fractional winding and the color-magnetic flux. In the presence of the $2 \mathrm{SC}$ condensate $\Phi_{2 \mathrm{SC}}$, the unbroken symmetry is $\tilde{H}_{2 \mathrm{SC}}=\mathrm{SU}(2)_{\mathrm{C}} \times \mathrm{U}(1)_{\mathrm{C}+\mathrm{B}}$ as in eq. (2.22). Then, the $\Phi_{d d}$ condensation can be taken as

$$
\Phi_{d d}=\left(\begin{array}{ccc}
\Delta_{d d}^{\prime} & 0 & \left(\Phi_{d d}\right)_{13} \\
0 & \Delta_{d d}^{\prime} & \left(\Phi_{d d}\right)_{23} \\
\left(\Phi_{d d}\right)_{31} & \left(\Phi_{d d}\right)_{32} & \Delta_{d d}^{\prime \prime}
\end{array}\right)
$$

without loss of generality. The $\mathrm{U}(1)_{\mathrm{C}+\mathrm{B}}$ symmetry in eq. (2.23), keeping the $2 \mathrm{SC}$ conden-

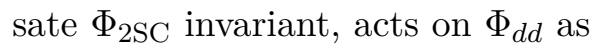

$$
\begin{aligned}
\Phi_{d d} & \rightarrow e^{2 i \alpha} e^{i \alpha T_{8}} \Phi_{d d}\left(e^{i \alpha T_{8}}\right)^{T} \\
& =\left(\begin{array}{ccc}
\Delta_{d d}^{\prime} & 0 & e^{3 i \alpha}\left(\Phi_{d d}\right)_{13} \\
0 & \Delta_{d d}^{\prime} & e^{3 i \alpha}\left(\Phi_{d d}\right)_{23} \\
e^{3 i \alpha}\left(\Phi_{d d}\right)_{31} & e^{3 i \alpha}\left(\Phi_{d d}\right)_{32} & e^{6 i \alpha} \Delta_{d d}^{\prime \prime}
\end{array}\right) .
\end{aligned}
$$

The minimal vortices depends on whether the off-diagonal blocks $\left(\Phi_{d d}\right)_{13},\left(\Phi_{d d}\right)_{23},\left(\Phi_{d d}\right)_{31}$, and $\left(\Phi_{d d}\right)_{32}$ are present or not. When the off-diagonal components vanish in the deconfined phase, the condensates on the angular coordinate $\varphi$ can be taken as $\varphi=6 \alpha$ as the minimal winding vortex, as discussed in the previous paper [77]. We call such a vortex as $\mathrm{U}(1)_{\mathrm{C}+\mathrm{B}}$ vortex (d). This is nothing but the Alice string locked with the $2 \mathrm{SC}$ condensate $\Phi_{2 \mathrm{SC}}$ in the deconfined phase.

Here, we restrict ourselves to the confined phase in which the off-diagonal blocks are present as in section 2.5. In this case, as the minimally winding vortex, we take the dependence of the condensates on the angular coordinate $\varphi$ as $\varphi=3 \alpha$ in eq. (5.4) for singlevaluedness of the off-diagonal components. We call such a vortex as $\mathrm{U}(1)_{\mathrm{C}+\mathrm{B}}$ vortex (c). We thus have an ansatz,

$$
\begin{aligned}
\Phi_{2 \mathrm{SC}}^{\alpha} & =\left(\begin{array}{lll}
0 & 0 & \Delta_{2 \mathrm{SC}}
\end{array}\right)^{T}, \\
\Phi_{d d}(\varphi) & =e^{2 i \varphi / 3} U(\varphi) \Phi_{d d}(0) U^{T}(\varphi) \\
& =\Delta_{d d}\left(\begin{array}{ccc}
g(r) & 0 & h_{1}(r) e^{i \varphi} \\
0 & g(r) & h_{2}(r) e^{i \varphi} \\
h_{1}(r) e^{i \varphi} & h_{2}(r) e^{i \varphi} & f(r) e^{i 2 \varphi}
\end{array}\right), \\
U(\varphi) & =e^{i(\varphi / 3) \operatorname{diag}(-1,-1,2)} \\
A_{i} & =-\frac{a(r)}{3 g} \frac{\epsilon_{i j} x^{j}}{r^{2}} \operatorname{diag}(-1,-1,2)
\end{aligned}
$$

where $f, g, h_{1}, h_{2}$ are profile functions with the boundary conditions

$$
\begin{aligned}
& f(0)=g^{\prime}(0)=h_{1}(0)=h_{2}(0)=0, \\
& f(\infty)=g(\infty)=h_{1}(\infty)=h_{2}(\infty)=1 .
\end{aligned}
$$


Here, we have set the condensate at $\varphi=0$ as

$$
\Phi_{d d}(\varphi=0)=\Phi_{d d}\left(\begin{array}{ccc}
g(r) & 0 & h_{1}(r) \\
0 & g(r) & h_{2}(r) \\
h_{1}(r) & h_{2}(r) & f(r)
\end{array}\right) .
$$

This carries $1 / 3$ quantized color-magnetic flux $\mathcal{F}_{0}$ and $2 / 3$ quantized circulation in $\mathrm{U}(1)_{\mathrm{B}}$. This is precisely a doubly-wound non-Abelian string locked with $\Phi_{2 \mathrm{SC}}$, which can be also understood as a mesonic bound state of two Alice strings. An interesting fact is that, in the confined phase, the $\mathrm{U}(1)_{\mathrm{C}+\mathrm{B}}$ vortex with $1 / 6$ flux cannot solely be observed but only the $\mathrm{U}(1)_{\mathrm{C}+\mathrm{B}}$ vortex with $1 / 3$ flux is allowed, unlike the deconfined phase allowing a $\mathrm{U}(1)_{\mathrm{C}+\mathrm{B}}$ vortex with $1 / 6$ flux. This difference can be understood from the order parameter manifolds in eq. (2.27).

For each of the above-mentioned vortices other typical configurations are given by the ones in eqs. (3.4), (3.5) and (3.6) by $r, g$, and $b$, respectively. These three configurations can be obtained by the color rotation, only together with the rotation of the $2 \mathrm{SC}$ condensate $\Phi_{2 \mathrm{SC}}$ in the color space.

\section{Summary and discussions}

We have proposed a novel confinement mechanism in the two-flavor dense quark matter that has confined and deconfined phases of vortices. As shown in the previous paper [77], the most stable vortices in the deconfined phase are non-Abelian Alice strings, which are superfluid vortices with non-Abelian color magnetic fluxes therein, exhibiting color nonsinglet $\mathrm{AB}$ phases. When the $2 \mathrm{SC}$ condensate $\Phi_{2 \mathrm{SC}}$ develops VEVs in the confined phase, it exhibits nontrivial (generalized) AB phases depending on the color around the nonAbelian Alice string. In the deconfined phase, this leads to the moduli locking; the 2SC condensate $\Phi_{2 S C}$ and the vortex moduli $\mathbb{R} P^{2}$ are locked [77]. On the other hand, in this paper, we have concentrated on the confined phase and have shown that vortices exhibiting color non-singlet $\mathrm{AB}$ phases are confined by the so-called $\mathrm{AB}$ defects to form color-singlet bound states. More precisely, it is inevitable that the Alice string is attached by the AB defect appearing to compensate the $\mathrm{AB}$ phase to maintain the singlevaluedness of the $2 \mathrm{SC}$ condensate $\Phi_{2 \mathrm{SC}}$. We have shown two possibilities of color singlet states as the fate of confinement; non-Abelian Alice strings are confined to either a baryonic or mesonic bound state in which constituent vortices are connected by AB defects. The baryonic bound state consists of three non-Abelian Alice strings with different color magnetic fluxes with the total flux canceled out, which are connected by a domain wall junction, while the mesonic bound state consists of two non-Abelian Alice strings with the same color magnetic fluxes. Although the latter contains a color magnetic flux in its core, this is already confined in the sense that it has only a color-singlet AB phase.

Several discussions are in order. In this paper, we have studied the confined phase, while the deconfined phase was studied in the previous paper [77]. In order to determine the phase diagram depending on the magnetic field, temperature and so on, we need for instance the Ginzburg-Landau (GL) theory of the two-flavor dense quark matter that 
remains as future problem. We have discussed a novel confinement mechanism of vortices in this paper. On the other hand, this $2 \mathrm{SC}+\langle d d\rangle$ phase also admits magnetic monopoles. Probably these monopoles are also confined as discussed in the SU(2) toy model [21] for which monopoles are twisted Alice strings and confined by $\mathrm{AB}$ defects. If monopoles are confined, this may show a duality between quark matter and hadronic matter; monopoles are confined where quarks are condensed in quark matter while quarks are confined where monopoles are condensed in hadron matter, analogous to the CFL phase [93].

Thus far, we have assumed that eigenvalues of $\langle d d\rangle$ are degenerate. In general, however, they do not have to be degenerate. They should be determined in the ground state, for instance, by the Ginzburg-Landau theory. For non-degenerate eigenvalues of $\langle d d\rangle$, the symmetry breaking pattern and possible vortex states are different.

We have neglected the electromagnetism in this paper. In the case of the CFL phase, the electromagnetic interaction induces the effective potential on the $\mathbb{C} P^{2}$ moduli space of a non-Abelian string [94]. A similar potential may exist on the $\mathbb{R} P^{2}$ moduli space of Alice string in the case of the $2 \mathrm{SC}+\langle d d\rangle$ phase as well.

Gapless fermions may exist in non-Abelian Alice strings in the $2 \mathrm{SC}+\langle d d\rangle$ as gapless Majorana fermion modes exist in non-Abelian vortices in the CFL phase [67, 68]. If they do, these modes may affect the confinement problem. Also, the gapless Majorana fermions trapped inside vortices endow a non-Abelian exchange statistics to them, thereby turning them into non-Abelian anyons [95], as is the case of non-Abelian vortices in the CFL phase $[96,97]$. It is interesting to study whether individual non-Abelian Alice strings as well as baryon and mesonic bound states are non-Abelian anyons before and after the vortex confinement, respectively.

Our analysis in this paper relies on the AB phase around vortices. This work focues on the confined phase in which color gauge group is completely broken by the both 2SC condensate and dd condensate. As all the gauge bosons are Higgs, gauge fluctuations are small at large distance, so that our $\mathrm{AB}$ phase works well in this set up. It should be noted, however, in the deconfined phase of the preceding work, there remains a $U(1)$ gauge symmetry; so it is a Coulomb phase. In this phase, the AB phase may not be described well by the classical treatment, we may need to take into account the correction due to gauge flucutuations, which will be left for the future research.

Finally, the confinement/deconfinement phase transition may be described in terms of higher-form symmetries (generalized global symmetries) [98], which is an indispensable tool to characterize the so-called topological order. In the CFL phase, higher form symmetries in the presence of non-Abelian semi-superfluid vortices were studied in refs. [35, 36, 72, 73, 99], in which a linking between a Wilson loop and a non-Abelian semi-superfluid vortex are all color singlet. Thus, the confinement and deconfinement phases of vortices may be distinguished by the higher form symmetry and associated topological order.

\section{Acknowledgments}

We thank Shigehiro Yasui for a discussion at the early stage of this work. This work is supported in part by Grant-in-Aid for Scientific Research, JSPS KAKENHI Grant Numbers 20J10506 (YF) and 18H01217 (MN). 


\section{A Pure color flux tubes}

Here, we summarize a color-magnetic flux tube generated only by the color gauge group $\mathrm{SU}(3)_{\mathrm{C}}$ without using the baryon symmetry $\mathrm{U}(1)_{\mathrm{B}}$, which is a local vortex. It is, however, unstable to decay into the ground state because of the trivial first homotopy group $\pi_{1}\left[\mathrm{SU}(3)_{\mathrm{C}}\right]=0$, as the same as color flux tubes discussed in the cases of the $2 \mathrm{SC}$ and CFL phases in refs. [76, 100], respectively. It is given by a closed loop in the group manifold $\mathrm{SU}(3)_{\mathrm{C}}$ as

$$
\begin{aligned}
\Phi_{d d}(\varphi) & =\Delta_{d d}\left(\begin{array}{ccc}
f(r) e^{-2 i \varphi} & 0 & 0 \\
0 & f(r) e^{-2 i \varphi} & 0 \\
0 & 0 & f(r) e^{4 i \varphi}
\end{array}\right), \\
A_{i} & =-\frac{a(r)}{g} \frac{\epsilon_{i j} x^{j}}{r^{2}} \operatorname{diag}(-1,-1,2),
\end{aligned}
$$

where the boundary conditions for the profile functions $f$ and $a$ are

$$
f(0)=a(0)=0, \quad f(\infty)=a(\infty)=1 .
$$

This has a color-magnetic flux

$$
\int d^{2} x F_{12}=\frac{2 \pi}{g} \operatorname{diag}(-1,-1,2)=\mathcal{F}_{0} \operatorname{diag}(-1,-1,2),
$$

where $F_{12}$ is the color-magnetic field strength tensor and a unit color-magnetic flux $\mathcal{F}_{0}$ is defined as

$$
\mathcal{F}_{0} \equiv \frac{2 \pi}{g}
$$

\section{B Generalized Aharonov-Bohm phases around vortices}

Here, we describe how to calculate (generalized) AB phases of light quarks, 2SC condensation, and heavy quarks around an Abelian superfluid string, pure color flux tube, non-Abelian Alice string, and doubly-wound non-Abelian string. Although these were already obtained in the previous paper [77] except for those of the doubly-wound non-Abelian string, we summarize them for this paper to be self-contained.

\section{B.1 Aharonov-Bohm phase around Abelian superfluid strings}

Abelian superfluid vortices have $B=1$ and $\mathcal{F}=0$, and let us substitute these into eq. (3.17). The generalized $\mathrm{AB}$ phases $\Gamma$ of light $(u, d)$ or heavy $(s)$ quarks encircling an Abelian $\mathrm{U}(1)_{\mathrm{B}}$ vortex can be summarized, by using short hand notation, as

$$
\begin{aligned}
& \begin{array}{llll}
r & g & b
\end{array} \\
& \Gamma_{\beta}^{u, d}(\varphi)=\left(\begin{array}{lll}
e^{+i \varphi / 2} & e^{+i \varphi / 2} & e^{+i \varphi / 2}
\end{array}\right) \\
& \Gamma_{\beta}^{s}(\varphi)=\left(\begin{array}{lll}
1 & 1 & 1
\end{array}\right),
\end{aligned}
$$


respectively, where the columns $(\beta=r, g, b)$ denote the colors of the light $(u, d)$ or heavy $(s)$ quarks encircling the vortex. Here, $\varphi$ is an azimuthal angle around the vortex. After the complete encirclement $\varphi=2 \pi$, these phases become

$$
\begin{aligned}
& \Gamma_{\beta}^{u, d}(\varphi=2 \pi)=\left(\begin{array}{ll}
-1-1 & -1)
\end{array},\right. \\
& \Gamma_{\beta}^{s}(\varphi=2 \pi)=(+1+1+1) .
\end{aligned}
$$

Thus, the light quarks receive generalized $\mathrm{AB}$ phases originating from vortex winding since they participate in the condensation with the vortex, while the heavy quark receive no phase in the absence of a color flux.

On the other hand, when the 2SC operator $\hat{\Phi}_{2 \mathrm{SC}}$ encircles the vortex, its generalized $\mathrm{AB}$ phases are

$$
\Gamma_{\alpha \beta}^{2 \mathrm{SC}}(\varphi)=\left(e^{+i \varphi} e^{+i \varphi} e^{+i \varphi}\right) .
$$

After the complete encirclement $\varphi=2 \pi$, these phases become

$$
\Gamma_{\alpha \beta}^{2 \mathrm{SC}}(\varphi=2 \pi)=(+1+1+1) .
$$

As expected, the generalized $\mathrm{AB}$ phases are all color-singlet since the Abelian vortex contains no color flux.

\section{B.2 Aharonov-Bohm phase around pure color flux tubes}

A pure color flux tube introduced in appendix A is generated by only color gauge symmetry and thus is unstable by the trivial homotopy group $\pi_{1}\left[\mathrm{SU}(3)_{\mathrm{C}}\right]=0$. Nevertheless we discuss $\mathrm{AB}$ phases around them because of usefulness for comparison with other topologically stable vortices.

\section{B.2.1 Pure color flux tube(d)}

In the deconfined phase, the pure color flux tube connects the two center elements 1 and $\omega^{2}$ of $\mathrm{SU}(3)_{\mathrm{C}}$ and thus carries a half $\mathrm{SU}(3)_{\mathrm{C}}$ flux: $B=0$ and $\mathcal{F}=1 / 2$. The asymptotic gauge fields of a color flux with a color $r, g, b$ are given by $A_{i}^{r} \propto \operatorname{diag}(2,-1,-1)$, $A_{i}^{g} \propto \operatorname{diag}(-1,-2,-1), A_{i}^{b} \propto \operatorname{diag}(-1,-1,2)$, respectively. Therefore, the AB phases of light $(u, d)$ or heavy $(s)$ quarks encircling flux tubes can be summarized as

$$
\Gamma_{\alpha \beta}^{u, d, s}(\varphi)=\left(\begin{array}{ccc}
e^{+i \varphi} & e^{-i \varphi / 2} & e^{-i \varphi / 2} \\
e^{-i \varphi / 2} & e^{+i \varphi} & e^{-i \varphi / 2} \\
e^{-i \varphi / 2} & e^{-i \varphi / 2} & e^{+i \varphi}
\end{array}\right)
$$

where the row $(\alpha=r, g, b)$ denotes the color of the flux tubes, and the column $(\beta=r, g, b)$ denotes the colors of the light $(u, d)$ or heavy $(s)$ quarks encircling them. After the complete encirclement $\varphi=2 \pi$, these phases become

$$
\Gamma_{\alpha \beta}^{u, d, s}(\varphi=2 \pi)=\left(\begin{array}{lll}
+1 & -1 & -1 \\
-1 & +1 & -1 \\
-1 & -1 & +1
\end{array}\right),
$$

which are color non-singlet. 
On the other hand, when the 2SC condensate operator $\hat{\Phi}_{2 \mathrm{SC}}$ encircles the flux tube, its $\mathrm{AB}$ phases are

$$
\Gamma_{\alpha \beta}^{2 \mathrm{SC}}(\varphi)=\left(\begin{array}{ccc}
e^{-i \varphi} & e^{+i \varphi / 2} & e^{+i \varphi / 2} \\
e^{+i \varphi / 2} & e^{-i \varphi} & e^{+i \varphi / 2} \\
e^{+i \varphi / 2} & e^{+i \varphi / 2} & e^{-i \varphi}
\end{array}\right) .
$$

After the complete encirclement $\varphi=2 \pi$, these phases become

$$
\Gamma_{\alpha \beta}^{2 \mathrm{SC}}(\varphi=2 \pi)=\left(\begin{array}{lll}
+1 & -1 & -1 \\
-1 & +1 & -1 \\
-1 & -1 & +1
\end{array}\right),
$$

which are color non-singlet as well.

The $\mathrm{AB}$ phase of the $u, d, s$ quarks and the $2 \mathrm{SC}$ condensate $\Phi_{2 \mathrm{SC}}$ are different among the colors, so they are color non-singlet.

\section{B.2.2 Pure color flux tube(c)}

In the confined phase, the pure color flux tube is generated by a closed loop in $\mathrm{SU}(3)_{\mathrm{C}}$ and thus carries the unit $\mathrm{SU}(3)_{\mathrm{C}}$ flux: $B=0$ and $\mathcal{F}=1$. The asymptotic gauge fields of a color flux with a color $r, g, b$ are the twice of those of the color flux in the deconfined phase. Therefore, the $\mathrm{AB}$ phases of the light $(u, d)$ or heavy $(s)$ quarks encircling flux tubes can be summarized as

$$
\Gamma_{\alpha \beta}^{u, d, s}(\varphi)=\left(\begin{array}{ccc}
e^{+2 i \varphi} & e^{-i \varphi} & e^{-i \varphi} \\
e^{-i \varphi} & e^{+2 i \varphi} & e^{-i \varphi} \\
e^{-i \varphi} & e^{-i \varphi} & e^{+2 i \varphi}
\end{array}\right)
$$

where the row $(\alpha=r, g, b)$ denotes the color of the flux tubes, and the column $(\beta=r, g, b)$ denotes the color of light $(u, d)$ or heavy $(s)$ quarks. After the complete encirclement $\varphi=2 \pi$, these phases become

$$
\Gamma_{\alpha \beta}^{u, d, s}(\varphi=2 \pi)=\left(\begin{array}{l}
+1+1+1 \\
+1+1+1 \\
+1+1+1
\end{array}\right),
$$

which are all color singlets.

On the other hand, when the $2 \mathrm{SC}$ operator $\hat{\Phi}_{2 \mathrm{SC}}$ encircles the flux tube, its $\mathrm{AB}$ phases are

$$
\Gamma_{\alpha \beta}^{2 \mathrm{SC}}(\varphi)=\left(\begin{array}{ccc}
e^{-2 i \varphi} & e^{+i \varphi} & e^{+i \varphi} \\
e^{+i \varphi} & e^{-2 i \varphi} & e^{+i \varphi} \\
e^{+i \varphi} & e^{+i \varphi} & e^{-2 i \varphi}
\end{array}\right) .
$$

After the complete encirclement $\varphi=2 \pi$, these phases become

$$
\Gamma_{\alpha \beta}^{2 \mathrm{SC}}(\varphi=2 \pi)=\left(\begin{array}{l}
+1+1+1 \\
+1+1+1 \\
+1+1+1
\end{array}\right)
$$

which are color singlet as well.

The AB phases of the $u, d, s$ quarks and the 2SC condensate operator $\hat{\Phi}_{2 \mathrm{SC}}$ are all color singlets. 


\section{B.3 Aharonov-Bohm phase around non-Abelian Alice strings}

A non-Abelian Alice string has $B=1 / 3$ and $\mathcal{F}=1 / 6$, and we thus substitute these into eq. (3.17), together with the asymptotic gauge fields of a color flux with a color $r, g, b$, given by $A_{i}^{r} \propto \operatorname{diag}(2,-1,-1), A_{i}^{g} \propto \operatorname{diag}(-1,-2,-1), A_{i}^{b} \propto \operatorname{diag}(-1,-1,2)$, respectively.

Therefore, the pure AB phases of heavy $(s)$ quark encircling flux tubes can be summarized, again by using short hand notation, as

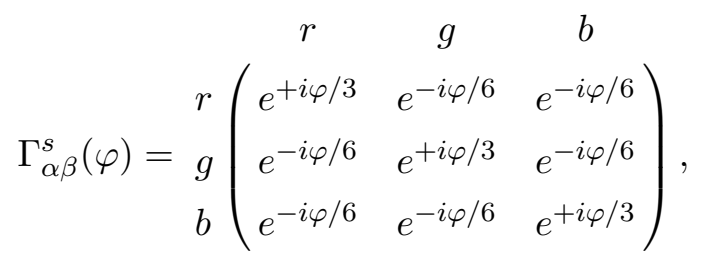

where, as explicitly indicated above, the row $(\alpha=r, g, b)$ denotes the color of the flux tubes, and the column $(\beta=r, g, b)$ denotes the colors of the heavy $(s)$ quark encircling them. After the complete encirclement $\varphi=2 \pi$, these phases become

$$
\Gamma_{\alpha \beta}^{s}(\varphi=2 \pi)=\left(\begin{array}{ccc}
\omega^{2} & \omega^{-1} & \omega^{-1} \\
\omega^{-1} & \omega^{2} & \omega^{-1} \\
\omega^{-1} & \omega^{-1} & \omega^{2}
\end{array}\right),
$$

which are color non-singlet. These form a $\mathbb{Z}_{6}$ group, and thus a set of the strange quarks come back to the original fields after complete encirclements of six times.

When the light quarks $u, d$ encircle the Alice string, they also receive $\mathrm{U}(1)_{\mathrm{B}}$ transformation $e^{+i \varphi / 6}$ as well as the $\mathrm{AB}$ phase that they have in common with those of the $s$-quarks. Therefore, generalized $\mathrm{AB}$ phases of the light quarks $u, d$ are given by

$$
\begin{aligned}
\Gamma_{\alpha \beta}^{u, d}(\varphi) & =e^{+i \varphi / 6} \Gamma_{\alpha \beta}^{s}(\varphi) \\
& =\left(\begin{array}{ccc}
e^{+i \varphi / 2} & 1 & 1 \\
1 & e^{+i \varphi / 2} & 1 \\
1 & 1 & e^{+i \varphi / 2}
\end{array}\right) .
\end{aligned}
$$

After the complete encirclement $\varphi=2 \pi$, these phases become

$$
\Gamma_{\alpha \beta}^{u, d}(\varphi=2 \pi)=\left(\begin{array}{l}
-1+1+1 \\
+1-1+1 \\
+1+1-1
\end{array}\right)
$$

which are a color non-singlet as well. We see that only quarks of the same color with that of the flux receive a nontrivial phase -1 .

On the other hand, when the 2SC operator $\hat{\Phi}_{2 \mathrm{SC}}$ encircles the Alice string, its generalized $\mathrm{AB}$ phases are

$$
\begin{aligned}
\Gamma_{\alpha \beta}^{2 \mathrm{SC}}(\varphi) & =e^{+i \varphi / 3} \Gamma_{\alpha \beta}^{s}(\varphi) \\
& =\left(\begin{array}{ccc}
1 & e^{+i \varphi / 2} & e^{+i \varphi / 2} \\
e^{+i \varphi / 2} & 1 & e^{+i \varphi / 2} \\
e^{+i \varphi / 2} & e^{+i \varphi / 2} & 1
\end{array}\right) .
\end{aligned}
$$


After the complete encirclement $\varphi=2 \pi$, these phases become

$$
\Gamma_{\alpha \beta}^{2 \mathrm{SC}}(\varphi=2 \pi)=\left(\begin{array}{lll}
+1 & -1 & -1 \\
-1 & +1 & -1 \\
-1 & -1 & +1
\end{array}\right)
$$

which are color non-singlet.

The (generalized) $\mathrm{AB}$ phases of the $u, d, s$ quarks and the $2 \mathrm{SC}$ operator $\hat{\Phi}_{2 \mathrm{SC}}$ are different among the colors, and so they are color non-singlet. Thus, one can read out the color of the flux from infinite distance by encircling the quarks or the $2 \mathrm{SC}$ condensate around the string at infinite distance.

This situation is in a sharp contrast to the case of the CFL phase, in which all (generalized) AB phases around non-Abelian vortices (color flux tubes) are color singlet [70, 71].

\section{B.4 Aharonov-Bohm phase around doubly-wound non-Abelian strings}

Substituting $B=2 / 3$ and $\Phi=1 / 3$ in eq. (3.17), one gets the followings. The pure $\mathrm{AB}$ phases of heavy $(s)$ quark encircling flux tubes can be summarized as

$$
\Gamma_{a b}^{s}(\varphi)=\left(\begin{array}{ccc}
e^{+2 i \varphi / 3} & e^{-i \varphi / 3} & e^{-i \varphi / 3} \\
e^{-i \varphi / 3} & e^{+2 i \varphi / 3} & e^{-i \varphi / 3} \\
e^{-i \varphi / 3} & e^{-i \varphi / 3} & e^{+2 i \varphi / 3}
\end{array}\right)
$$

where the row $(a=r, g, b)$ denotes the color of the flux tubes, and the column $(b=r, g, b)$ denotes the colors of the heavy $(s)$ quark encircling them. After the complete encirclement $\varphi=2 \pi$, these phases become

$$
\Gamma_{a b}^{s}(\varphi=2 \pi)=\left(\begin{array}{ccc}
\omega^{4} & \omega^{-2} & \omega^{-2} \\
\omega^{-2} & \omega^{4} & \omega^{-2} \\
\omega^{-2} & \omega^{-2} & \omega^{4}
\end{array}\right)=\omega^{4}\left(\begin{array}{c}
+1+1+1 \\
+1+1+1 \\
+1+1+1
\end{array}\right) .
$$

These are nonzero $\mathrm{AB}$ phases and are color singlets, forming a $\mathbb{Z}_{3}$ group.

When the light quarks $u, d$ encircle the doubly-wound non-Abelian string, they also receive a $\mathrm{U}(1)_{\mathrm{B}}$ transformation. Therefore, generalized $\mathrm{AB}$ phases of the light quarks $u, d$ are

$$
\begin{aligned}
\Gamma_{a b}^{u, d}(\varphi) & =e^{+i \varphi / 3} \Gamma_{a b}^{s}(\varphi) \\
& =e^{+i \varphi / 3}\left(\begin{array}{ccc}
e^{+2 i \varphi / 3} & e^{-i \varphi / 3} & e^{-i \varphi / 3} \\
e^{-i \varphi / 3} & e^{+2 i \varphi / 3} & e^{-i \varphi / 6} \\
e^{-i \varphi / 3} & e^{-i \varphi / 3} & e^{+2 i \varphi / 3}
\end{array}\right) \\
& =\left(\begin{array}{ccc}
e^{+i \varphi} & 1 & 1 \\
1 & e^{+i \varphi} & 1 \\
1 & 1 & e^{+i \varphi}
\end{array}\right) .
\end{aligned}
$$

After the complete encirclement $\varphi=2 \pi$, these phases become

$$
\Gamma_{a b}^{u, d}(\varphi=2 \pi)=\left(\begin{array}{l}
+1+1+1 \\
+1+1+1 \\
+1+1+1
\end{array}\right)
$$

which are color singlet. 
On the other hand, when the 2SC operator $\hat{\Phi}_{2 \mathrm{SC}}$ encircles the doubly-wound nonAbelian string, its generalized $\mathrm{AB}$ phases are

$$
\begin{aligned}
\Gamma_{\alpha \beta}^{2 \mathrm{SC}}(\varphi) & =e^{+2 i \varphi / 3}\left(\begin{array}{ccc}
e^{-2 i \varphi / 3} & e^{+i \varphi / 3} & e^{+i \varphi / 3} \\
e^{+i \varphi / 3} & e^{-2 i \varphi / 3} & e^{+i \varphi / 3} \\
e^{+i \varphi / 3} & e^{+i \varphi / 6} & e^{-2 i \varphi / 3}
\end{array}\right) \\
& =\left(\begin{array}{ccc}
1 & e^{+i \varphi} & e^{+i \varphi} \\
e^{+i \varphi} & 1 & e^{+i \varphi} \\
e^{+i \varphi} & e^{+i \varphi} & 1
\end{array}\right)
\end{aligned}
$$

After the complete encirclement $\varphi=2 \pi$, these phases become

$$
\Gamma_{\alpha \beta}^{2 \mathrm{SC}}(\varphi=2 \pi)=\left(\begin{array}{l}
+1+1+1 \\
+1+1+1 \\
+1+1+1
\end{array}\right)
$$

which are color singlet as well.

Thus, we conclude that the (generalized) $\mathrm{AB}$ phases of the $u, d, s$ quarks and the 2SC operator $\hat{\Phi}_{2 \mathrm{SC}}$ are all color singlets around the doubly-wound non-Abelian string.

In summary of this appendix, the (generalized) $\mathrm{AB}$ phases are all color singlet for the Abelian superfluid string and doubly-wound non-Abelian string, while these are color non-singlet around the non-Abelian Alice string. This fact indicates that an Abelian superfluid string and doubly-wound non-Abelian string can be present in the confined phase as discussed in the main text.

Open Access. This article is distributed under the terms of the Creative Commons Attribution License (CC-BY 4.0), which permits any use, distribution and reproduction in any medium, provided the original author(s) and source are credited.

\section{References}

[1] Y. Nambu, Strings, Monopoles and Gauge Fields, Phys. Rev. D 10 (1974) 4262 [InSPIRE].

[2] S. Mandelstam, Vortices and Quark Confinement in Nonabelian Gauge Theories, Phys. Rept. 23 (1976) 245 [inSPIRE].

[3] A.M. Polyakov, Quark Confinement and Topology of Gauge Groups, Nucl. Phys. B 120 (1977) 429 [INSPIRE].

[4] D.T. Son and M.A. Stephanov, Domain walls in two-component Bose-Einstein condensates, Phys. Rev. A 65 (2002) 063621 [cond-mat/0103451] [INSPIRE].

[5] K. Kasamatsu, M. Tsubota and M. Ueda, Vortex molecules in coherently coupled two-component Bose-Einstein condensates, Phys. Rev. Lett. 93 (2004) 250406 [cond-mat/0406150] [INSPIRE].

[6] M. Cipriani and M. Nitta, Crossover between integer and fractional vortex lattices in coherently coupled two-component Bose-Einstein condensates, Phys. Rev. Lett. 111 (2013) 170401 [arXiv: 1303.2592] [INSPIRE]. 
[7] M. Tylutki, L.P. Pitaevskii, A. Recati and S. Stringari, Confinement and precession of vortex pairs in coherently coupled Bose-Einstein condensates, Phys. Rev. A 93 (2016) 043623 [arXiv: 1601.03695] [INSPIRE].

[8] M. Eto and M. Nitta, Confinement of half-quantized vortices in coherently coupled Bose-Einstein condensates: Simulating quark confinement in a QCD-like theory, Phys. Rev. A 97 (2018) 023613 [arXiv: 1702.04892] [INSPIRE].

[9] M. Eto, K. Ikeno and M. Nitta, Collision dynamics and reactions of fractional vortex molecules in coherently coupled Bose-Einstein condensates, Phys. Rev. Res. 2 (2020) 033373 [arXiv: 1912.09014] [INSPIRE].

[10] M. Kobayashi, M. Eto and M. Nitta, Berezinskii-Kosterlitz-Thouless Transition of Two-Component Bose Mixtures with Intercomponent Josephson Coupling, Phys. Rev. Lett. 123 (2019) 075303 [arXiv: 1802.08763] [INSPIRE].

[11] E. Babaev, Vortices carrying an arbitrary fraction of magnetic flux quantum in two gap superconductors, Phys. Rev. Lett. 89 (2002) 067001 [cond-mat/0111192] [INSPIRE].

[12] Y. Tanaka, Phase instability in multi-band superconductors, J. Phys. Soc. Jap. 70 (2001) 2844.

[13] Y. Tanaka, Soliton in two-band superconductor, Phys. Rev. Lett. 88 (2001) 017002.

[14] J. Goryo, S. Soma and H. Matsukawa, Deconfinement of vortices with continuously variable fractions of the unit flux quanta in two-gap superconductors, Europhys. Lett. 80 (2007) 17002.

[15] M. Shifman and M. Ünsal, QCD-like Theories on $R_{3} \times S_{1}$ : A Smooth Journey from Small to Large $r\left(S_{1}\right)$ with Double-Trace Deformations, Phys. Rev. D 78 (2008) 065004 [arXiv:0802.1232] [INSPIRE].

[16] D.B. Carpenter and J.T. Chalker, The phase diagram of a generalised XY model, J. Phys. Condens. Matter 1 (1989) 4907.

[17] M. Kobayashi, G. Fejös, C. Chatterjee and M. Nitta, Vortex confinement transitions in the modified Goldstone model, Phys. Rev. Res. 2 (2020) 013081 [arXiv:1908.11087] [InSPIRE].

[18] M. Kobayashi and M. Nitta, $\mathbb{Z}_{n}$ modified $X Y$ and Goldstone models and vortex confinement transition, Phys. Rev. D 101 (2020) 085003 [arXiv: 1912.09456] [InSPIRE].

[19] C. Chatterjee and M. Nitta, Aharonov-Bohm defects, Phys. Rev. D 101 (2020) 085002 [arXiv:1905.01884] [INSPIRE].

[20] C. Chatterjee, M. Kurachi and M. Nitta, Topological Defects in the Georgi-Machacek Model, Phys. Rev. D 97 (2018) 115010 [arXiv:1801.10469] [InSPIRE].

[21] M. Nitta, Confinement and moduli locking of Alice strings and monopoles, JHEP 03 (2021) 276 [arXiv:2011.14396] [INSPIRE].

[22] D. Bailin and A. Love, Superfluidity and Superconductivity in Relativistic Fermion Systems, Phys. Rept. 107 (1984) 325 [INSPIRE].

[23] M. Iwasaki and T. Iwado, Superconductivity in the quark matter, Phys. Lett. B 350 (1995) 163 [INSPIRE].

[24] M.G. Alford, A. Schmitt, K. Rajagopal and T. Schäfer, Color superconductivity in dense quark matter, Rev. Mod. Phys. 80 (2008) 1455 [arXiv:0709.4635] [INSPIRE]. 
[25] M.G. Alford, K. Rajagopal and F. Wilczek, Color flavor locking and chiral symmetry breaking in high density QCD, Nucl. Phys. B 537 (1999) 443 [hep-ph/9804403] [INSPIRE].

[26] M.G. Alford, K. Rajagopal and F. Wilczek, QCD at finite baryon density: Nucleon droplets and color superconductivity, Phys. Lett. B 422 (1998) 247 [hep-ph/9711395] [INSPIRE].

[27] R. Rapp, T. Schäfer, E.V. Shuryak and M. Velkovsky, Diquark Bose condensates in high density matter and instantons, Phys. Rev. Lett. 81 (1998) 53 [hep-ph/9711396] [INSPIRE].

[28] T. Schäfer and F. Wilczek, Continuity of quark and hadron matter, Phys. Rev. Lett. 82 (1999) 3956 [hep-ph/9811473] [INSPIRE].

[29] M.G. Alford, J. Berges and K. Rajagopal, Unlocking color and flavor in superconducting strange quark matter, Nucl. Phys. B 558 (1999) 219 [hep-ph/9903502] [INSPIRE].

[30] K. Fukushima, Quark description of the Nambu-Goldstone bosons in the color flavor locked phase, Phys. Rev. D 70 (2004) 094014 [hep-ph/0403091] [INSPIRE].

[31] T. Hatsuda, M. Tachibana, N. Yamamoto and G. Baym, New critical point induced by the axial anomaly in dense QCD, Phys. Rev. Lett. 97 (2006) 122001 [hep-ph/0605018] [INSPIRE].

[32] N. Yamamoto, M. Tachibana, T. Hatsuda and G. Baym, Phase structure, collective modes, and the axial anomaly in dense QCD, Phys. Rev. D 76 (2007) 074001 [arXiv:0704.2654] [INSPIRE].

[33] T. Hatsuda, M. Tachibana and N. Yamamoto, Spectral Continuity in Dense QCD, Phys. Rev. D 78 (2008) 011501 [arXiv:0802.4143] [INSPIRE].

[34] A. Schmitt, S. Stetina and M. Tachibana, Ginzburg-Landau phase diagram for dense matter with axial anomaly, strange quark mass, and meson condensation, Phys. Rev. D 83 (2011) 045008 [arXiv: 1010.4243 ] [INSPIRE].

[35] A. Cherman, S. Sen and L.G. Yaffe, Anyonic particle-vortex statistics and the nature of dense quark matter, Phys. Rev. D 100 (2019) 034015 [arXiv:1808.04827] [INSPIRE].

[36] A. Cherman, T. Jacobson, S. Sen and L.G. Yaffe, Higgs-confinement phase transitions with fundamental representation matter, Phys. Rev. D 102 (2020) 105021 [arXiv:2007.08539] [INSPIRE].

[37] K. Masuda, T. Hatsuda and T. Takatsuka, Hadron-Quark Crossover and Massive Hybrid Stars with Strangeness, Astrophys. J. 764 (2013) 12 [arXiv:1205.3621] [INSPIRE].

[38] K. Masuda, T. Hatsuda and T. Takatsuka, Hadron-quark crossover and massive hybrid stars, PTEP 2013 (2013) 073D01 [arXiv:1212.6803] [INSPIRE].

[39] T. Kojo, P.D. Powell, Y. Song and G. Baym, Phenomenological QCD equation of state for massive neutron stars, Phys. Rev. D 91 (2015) 045003 [arXiv:1412.1108] [INSPIRE].

[40] G. Baym, T. Hatsuda, T. Kojo, P.D. Powell, Y. Song and T. Takatsuka, From hadrons to quarks in neutron stars: a review, Rept. Prog. Phys. 81 (2018) 056902 [arXiv:1707.04966] [INSPIRE].

[41] G. Baym, S. Furusawa, T. Hatsuda, T. Kojo and H. Togashi, New Neutron Star Equation of State with Quark-Hadron Crossover, Astrophys. J. 885 (2019) 42 [arXiv:1903.08963] [INSPIRE]. 
[42] Y. Fujimoto, K. Fukushima and W. Weise, Continuity from neutron matter to two-flavor quark matter with ${ }^{1} S_{0}$ and ${ }^{3} P_{2}$ superfluidity, Phys. Rev. D 101 (2020) 094009 [arXiv: 1908.09360] [INSPIRE].

[43] Y. Fujimoto, Continuity from neutron matter to color-superconducting quark matter with ${ }^{3} P_{2}$ superfluidity, Nucl. Phys. A 1005 (2021) 121757 [arXiv:2002.08073] [INSPIRE].

[44] M. Hoffberg, A.E. Glassgold, R.W. Richardson and M. Ruderman, Anisotropic Superfluidity in Neutron Star Matter, Phys. Rev. Lett. 24 (1970) 775 [INSPIRE].

[45] R. Tamagaki, Superfluid State in Neutron Star Matter. I. Generalized Bogoliubov Transformation and Existence of ${ }^{3} \mathrm{P}_{2}$ Gap at High Density, Prog. Theor. Phys. 44 (1970) 905.

[46] T. Takatsuka and R. Tamagaki, Superfluid State in Neutron Star Matter. II: Properties of Anisotropic Energy Gap of ${ }^{3} P_{2}$ Pairing, Prog. Theor. Phys. 46 (1971) 114.

[47] T. Takatsuka, Superfluid State in Neutron Star Matter. III: Tensor Coupling Effect in ${ }^{3} P_{2}$ Energy Gap, Prog. Theor. Phys. 47 (1972) 1062.

[48] R.W. Richardson, Ginzburg-Landau theory of anisotropic superfluid neutron-star matter, Phys. Rev. D 5 (1972) 1883 [inSPIRE].

[49] J. Sauls and J. Serene, ${ }^{3} P_{2}$ pairing near the transition temperature in neutron-star matter, Phys. Rev. D 17 (1978) 1524 [InSPIRE].

[50] T. Takatsuka and R. Tamagaki, Superfluidity in neutron star matter and symmetric nuclear matter, Prog. Theor. Phys. Suppl. 112 (1993) 27 [InSPIRE].

[51] K. Masuda and M. Nitta, Magnetic Properties of Quantized Vortices in Neutron ${ }^{3} P_{2}$ Superfluids in Neutron Stars, Phys. Rev. C 93 (2016) 035804 [arXiv:1512.01946] [INSPIRE].

[52] T. Mizushima, K. Masuda and M. Nitta, ${ }^{3} P_{2}$ superfluids are topological, Phys. Rev. B 95 (2017) 140503 [arXiv: 1607.07266] [inSPIRE].

[53] S. Yasui, C. Chatterjee and M. Nitta, Phase structure of neutron ${ }^{3} P_{2}$ superfluids in strong magnetic fields in neutron stars, Phys. Rev. C 99 (2019) 035213 [arXiv:1810.04901] [INSPIRE].

[54] S. Yasui, C. Chatterjee, M. Kobayashi and M. Nitta, Reexamining Ginzburg-Landau theory for neutron ${ }^{3} P_{2}$ superfluidity in neutron stars, Phys. Rev. C 100 (2019) 025204 [arXiv: 1904.11399] [INSPIRE].

[55] T. Mizushima, S. Yasui and M. Nitta, Critical end point and universality class of neutron ${ }^{3} P_{2}$ superfluids in neutron stars, Phys. Rev. Res. 2 (2020) 013194 [arXiv:1908.07944] [INSPIRE].

[56] M. Eto, Y. Hirono, M. Nitta and S. Yasui, Vortices and Other Topological Solitons in Dense Quark Matter, PTEP 2014 (2014) 012D01 [arXiv: 1308.1535] [INSPIRE].

[57] M.M. Forbes and A.R. Zhitnitsky, Global strings in high density QCD, Phys. Rev. D 65 (2002) 085009 [hep-ph/0109173] [INSPIRE].

[58] K. Iida and G. Baym, Superfluid phases of quark matter. 3. Supercurrents and vortices, Phys. Rev. D 66 (2002) 014015 [hep-ph/0204124] [InSPIRE].

[59] E. Nakano, M. Nitta and T. Matsuura, Non-Abelian strings in high density QCD: Zero modes and interactions, Phys. Rev. D 78 (2008) 045002 [arXiv:0708.4096] [INSPIRE]. 
[60] M. Cipriani, W. Vinci and M. Nitta, Colorful boojums at the interface of a color superconductor, Phys. Rev. D 86 (2012) 121704 [arXiv: 1208.5704] [INSPIRE].

[61] M.G. Alford, S.K. Mallavarapu, T. Vachaspati and A. Windisch, Stability of superfluid vortices in dense quark matter, Phys. Rev. C 93 (2016) 045801 [arXiv:1601.04656] [INSPIRE].

[62] A.P. Balachandran, S. Digal and T. Matsuura, Semi-superfluid strings in high density QCD, Phys. Rev. D 73 (2006) 074009 [hep-ph/0509276] [INSPIRE].

[63] E. Nakano, M. Nitta and T. Matsuura, Non-Abelian Strings in Hot or Dense QCD, Prog. Theor. Phys. Suppl. 174 (2008) 254 [arXiv:0805.4539] [INSPIRE].

[64] M. Eto and M. Nitta, Color Magnetic Flux Tubes in Dense QCD, Phys. Rev. D 80 (2009) 125007 [arXiv: 0907.1278] [INSPIRE].

[65] M. Eto, E. Nakano and M. Nitta, Effective world-sheet theory of color magnetic flux tubes in dense QCD, Phys. Rev. D 80 (2009) 125011 [arXiv:0908.4470] [InSPIRE].

[66] M. Eto, M. Nitta and N. Yamamoto, Instabilities of Non-Abelian Vortices in Dense QCD, Phys. Rev. Lett. 104 (2010) 161601 [arXiv:0912.1352] [InSPIRE].

[67] S. Yasui, K. Itakura and M. Nitta, Fermion structure of non-Abelian vortices in high density QCD, Phys. Rev. D 81 (2010) 105003 [arXiv:1001.3730] [InSPIRE].

[68] T. Fujiwara, T. Fukui, M. Nitta and S. Yasui, Index theorem and Majorana zero modes along a non-Abelian vortex in a color superconductor, Phys. Rev. D 84 (2011) 076002 [arXiv:1105.2115] [INSPIRE].

[69] M.G. Alford, G. Baym, K. Fukushima, T. Hatsuda and M. Tachibana, Continuity of vortices from the hadronic to the color-flavor locked phase in dense matter, Phys. Rev. D 99 (2019) 036004 [arXiv: 1803.05115] [INSPIRE].

[70] C. Chatterjee, M. Nitta and S. Yasui, Quark-hadron continuity under rotation: Vortex continuity or boojum?, Phys. Rev. D 99 (2019) 034001 [arXiv: 1806.09291] [inSPIRE].

[71] C. Chatterjee, M. Nitta and S. Yasui, Quark-Hadron Crossover with Vortices, JPS Conf. Proc. 26 (2019) 024030 [arXiv: 1902.00156] [INSPIRE].

[72] Y. Hirono and Y. Tanizaki, Quark-Hadron Continuity beyond the Ginzburg-Landau Paradigm, Phys. Rev. Lett. 122 (2019) 212001 [arXiv:1811.10608] [INSPIRE].

[73] Y. Hirono and Y. Tanizaki, Effective gauge theories of superfluidity with topological order, JHEP 07 (2019) 062 [arXiv: 1904.08570] [INSPIRE].

[74] Y. Fujimoto and M. Nitta, Alice meets Boojums in neutron stars: vortices penetrating two-flavor quark-hadron continuity, Phys. Rev. D 103 (2021) 114003 [arXiv:2102.12928] [INSPIRE].

[75] M. Eto and M. Nitta, Chiral non-Abelian vortices and their confinement in three flavor dense $Q C D$, arXiv:2103.13011 [INSPIRE].

[76] M.G. Alford and A. Sedrakian, Color-magnetic flux tubes in quark matter cores of neutron stars, J. Phys. G 37 (2010) 075202 [arXiv:1001.3346] [INSPIRE].

[77] Y. Fujimoto and M. Nitta, Non-Abelian Alice strings in two-flavor dense QCD, Phys. Rev. D 103 (2021) 054002 [arXiv: 2011.09947] [INSPIRE].

[78] A.S. Schwarz, Field Theories with No Local Conservation of the Electric Charge, Nucl. Phys. B 208 (1982) 141 [INSPIRE]. 
[79] M.G. Alford, K. Benson, S.R. Coleman, J. March-Russell and F. Wilczek, The Interactions and Excitations of Nonabelian Vortices, Phys. Rev. Lett. 64 (1990) 1632 [Erratum ibid. 65 (1990) 668] [INSPIRE].

[80] M.G. Alford, K. Benson, S.R. Coleman, J. March-Russell and F. Wilczek, Zero modes of nonabelian vortices, Nucl. Phys. B 349 (1991) 414 [INSPIRE].

[81] M.G. Alford, K.-M. Lee, J. March-Russell and J. Preskill, Quantum field theory of nonAbelian strings and vortices, Nucl. Phys. B 384 (1992) 251 [hep-th/9112038] [INSPIRE].

[82] J. Preskill and L.M. Krauss, Local Discrete Symmetry and Quantum Mechanical Hair, Nucl. Phys. B 341 (1990) 50 [INSPIRE].

[83] M. Bucher, H.-K. Lo and J. Preskill, Topological approach to Alice electrodynamics, Nucl. Phys. B 386 (1992) 3 [hep-th/9112039] [InSPIRE].

[84] H.-K. Lo and J. Preskill, NonAbelian vortices and nonAbelian statistics, Phys. Rev. D 48 (1993) 4821 [hep-th/9306006] [INSPIRE].

[85] C. Chatterjee and M. Nitta, BPS Alice strings, JHEP 09 (2017) 046 [arXiv:1703.08971] [INSPIRE].

[86] C. Chatterjee and M. Nitta, The effective action of a BPS Alice string, Eur. Phys. J. C 77 (2017) 809 [arXiv: 1706.10212] [INSPIRE].

[87] M. Eto and M. Nitta, Vortex trimer in three-component Bose-Einstein condensates, Phys. Rev. A 85 (2012) 053645 [arXiv: 1201.0343] [INSPIRE].

[88] M. Nitta, M. Eto, T. Fujimori and K. Ohashi, Baryonic Bound State of Vortices in Multicomponent Superconductors, J. Phys. Soc. Jap. 81 (2012) 084711 [arXiv:1011.2552] [INSPIRE].

[89] R. Auzzi and M. Shifman, Low-Energy Limit of Yang-Mills with Massless Adjoint Quarks: Chiral Lagrangian and Skyrmions, J. Phys. A 40 (2007) 6221 [hep-th/0612211] [InSPIRE].

[90] R. Auzzi, S. Bolognesi and M. Shifman, Skyrmions in Yang-Mills Theories with Massless Adjoint Quarks, Phys. Rev. D 77 (2008) 125029 [arXiv:0804.0229] [INSPIRE].

[91] S. Bolognesi, C. Chatterjee and K. Konishi, NonAbelian Vortices, Large Winding Limits and Aharonov-Bohm Effects, JHEP 04 (2015) 143 [arXiv: 1503.00517] [INSPIRE].

[92] C. Chatterjee and M. Nitta, Aharonov-Bohm Phase in High Density Quark Matter, Phys. Rev. D 93 (2016) 065050 [arXiv: 1512.06603] [INSPIRE].

[93] M. Eto, M. Nitta and N. Yamamoto, Confined Monopoles Induced by Quantum Effects in Dense QCD, Phys. Rev. D 83 (2011) 085005 [arXiv:1101.2574] [INSPIRE].

[94] W. Vinci, M. Cipriani and M. Nitta, Spontaneous Magnetization through Non-Abelian Vortex Formation in Rotating Dense Quark Matter, Phys. Rev. D 86 (2012) 085018 [arXiv:1206.3535] [INSPIRE].

[95] D.A. Ivanov, Non-abelian statistics of half-quantum vortices in p-wave superconductors, Phys. Rev. Lett. 86 (2001) 268 [cond-mat/0005069] [INSPIRE].

[96] S. Yasui, K. Itakura and M. Nitta, Majorana meets Coxeter: Non-Abelian Majorana Fermions and Non-Abelian Statistics, Phys. Rev. B 83 (2011) 134518 [arXiv:1010.3331] [INSPIRE].

[97] Y. Hirono, S. Yasui, K. Itakura and M. Nitta, Non-Abelian statistics of vortices with multiple Majorana fermions, Phys. Rev. B 86 (2012) 014508 [arXiv:1203.0173] [INSPIRE]. 
[98] D. Gaiotto, A. Kapustin, N. Seiberg and B. Willett, Generalized Global Symmetries, JHEP 02 (2015) 172 [arXiv: 1412.5148] [INSPIRE].

[99] Y. Hidaka, Y. Hirono, M. Nitta, Y. Tanizaki and R. Yokokura, Topological order in the color-flavor locked phase of a $(3+1)$-dimensional $U(N)$ gauge-Higgs system, Phys. Rev. D 100 (2019) 125016 [arXiv: 1903.06389] [INSPIRE].

[100] K. Iida, Magnetic vortex in color-flavor locked quark matter, Phys. Rev. D 71 (2005) 054011 [hep-ph/0412426] [INSPIRE]. 